Arylsulfonyl fluoride boronic acids:

Leave this area blank for abstract info.

Preparation and coupling reactivity

Terry Shing-Bong Lou, and Michael C. Willis

Department of Chemistry, University of Oxford, Chemistry Research Laboratory, Mansfield Road, Oxford, OXI 3TA, UK

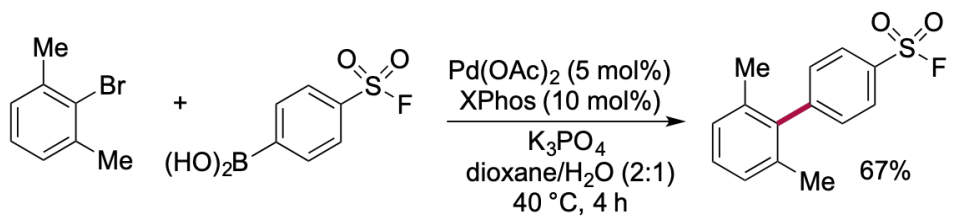




\title{
Arylsulfonyl fluoride boronic acids: Preparation and coupling reactivity
}

\author{
Terry Shing-Bong Lou, and Michael C. Willis* \\ Department of Chemistry, University of Oxford, Chemistry Research Laboratory, Mansfield Road, Oxford, OX1 3TA, UK.
}

This paper is dedicated to Professor Steve Davies in recognition of his contributions to organic chemistry

\section{ARTICLE INFO}

\section{ABSTRACT}

\section{Article history:}

Received

Received in revised form

Accepted

Available online

Keywords:

Boronic acid

Sulfonyl fluoride

Palladium

Rhodium

\begin{abstract}
We report the efficient and practical syntheses of ortho-, meta, and para-sulfonyl fluoride substituted benzene boronic acids. The syntheses of the para- and meta-isomers commence with the appropriate bromo-substituted benzenesulfonyl chlorides, and the ortho-isomer is prepared from benzenesulfonyl fluoride. The para- and meta-substituted boronic acids undergo efficient Sukuki-Miyaura coupling reactions with a range of aryl halides. We also report an efficient $\mathrm{Rh}(\mathrm{I})$ catalyzed conjugate addition reaction using the para-substituted boronic acid.
\end{abstract}

\section{Introduction}

The balance of reactivity and stability available in sulfonyl fluorides has made them popular motifs in medicinal chemistry ${ }^{1}$ and chemical biology. ${ }^{2}$ Their stability to physiological hydrolysis, their utility in SuFEx click chemistry, ${ }^{3}$ and the ability to act as fluorinating reagents, has resulted in a variety of applications. Scheme 1 illustrates this with examples of serine protease inhibitors (AEBSF, Pefabloc and PMSF), ${ }^{4}$ the probe reagent FBSA, ${ }^{5}$ and Doyle's PyFluor ${ }^{6}$ fluorinating reagent.

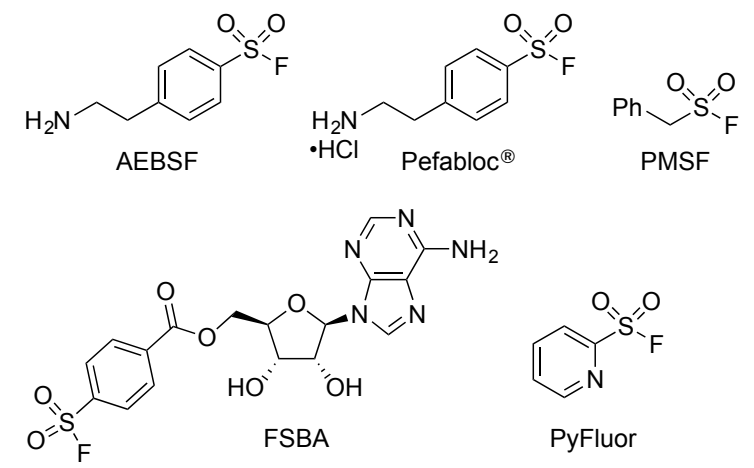

Scheme 1. Sulfonyl fluorides in medicinal chemistry, as chemical probes, and as fluorinating reagents.

The most common method to prepare sulfonyl fluorides is by chloride to fluoride exchange using the corresponding sulfonyl chlorides. These reactions typically use toxic potassium bifluoride, or potassium fluoride in combination with 18-crown-6. In addition, sulfonyl chlorides can often show limited stability, and can be challenging to prepare and purify. ${ }^{7}$ Sulfonates, and various sulfonate-derivatives such as sulfonylhydrazides, ${ }^{8}$ can also be transformed into sulfonyl flourides, although these approaches can be limited by the availability of the required substrates. Aryl halides and alkenyl triflates have been exploited as sulfonyl fluoride precursors via palladium-catalyzed sulfination chemistry, using sulfur dioxide surrogates, ${ }^{9}$ followed by electrophilic fluorination. ${ }^{10}$ Sulfonyl fluoride synthesis by the electrochemical oxidation of thiols has recently been reported. ${ }^{11}$

The chemical stability present in sulfonyl flourides has allowed alternative approaches to these motifs based on the functionalization of molecules containing a pre-existing sulfonyl fluoride functional group. A useful example of this is the use of ethenesulfonyl fluoride (ESF) in a variety of conjugate additiontype processes. ${ }^{12}$ The stability of sulfonyl fluorides has also been exploited in a number of transition metal-catalyzed reactions, and this chemistry has recently been reviewed by Chinthakindi and Arvidsson. ${ }^{13}$ Reaction 1 of Scheme 2 is illustrative, and shows ESF being employed as the alkene component in a Heck coupling with an aryl halide. ${ }^{14}$ Related Heck-type reactions using aryl boronic acids, ${ }^{15}$ and also aryl diazonium salts, ${ }^{16}$ both in combination with ESF and palladium catalysts have also been reported. Aryl halides substituted with sulfonyl fluorides have been used as crosscoupling partners in Suzuki-Miyaura reactions (reaction 2, Scheme 2), ${ }^{17}$ in Sonogashira coupling reactions (reaction 3, Scheme 2), ${ }^{2 \mathrm{a}}$ in Stille coupling, ${ }^{2 \mathrm{~d}}$ and in a Negishi cross-coupling reaction with diethylzinc. ${ }^{17 \mathrm{~b}}$ In addition to these palladiumcatalyzed processes, sulfonyl fluorides have also been shown to be 
tolerant to certain copper-, ${ }^{18}$ iridium-,${ }^{2 \mathrm{a}}$ and rhodium-catalyzed reactions. $^{19}$

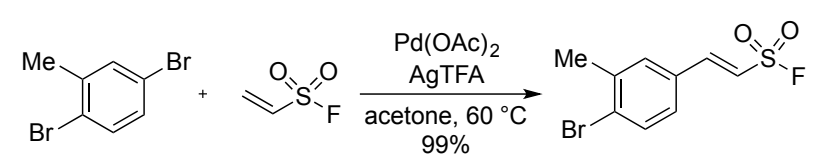

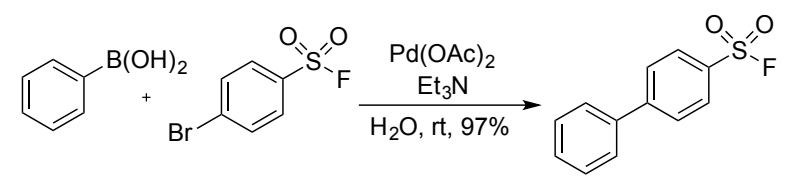

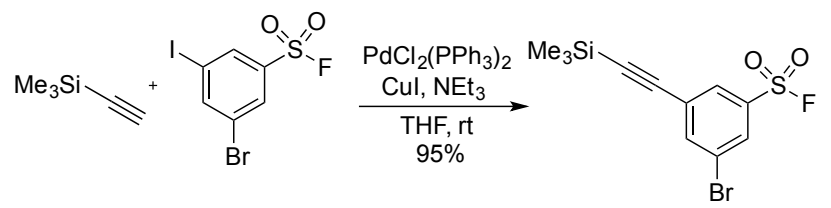

Scheme 2. Sulfonyl fluorides containing molecules in palladiumcatalyzed reactions.

Although sulfonyl fluoride-substituted aryl halides have been used in Suzuki-Miyaura cross-coupling reactions (reaction 1, Scheme 2), the corresponding sulfonyl fluoride-substituted aryl boronic acids have not been reported, although one example of a boronic ester is known. ${ }^{2 a}$ Given the tolerance of sulfonyl fluorides to a variety of palladium-catalyzed reactions, as well as other metal-mediated processes, together with the widespread use of Suzuki-Miyaura reactions in synthetic chemistry, we reasoned that such boronic acids would be useful reagents, and would add to the repertoire of methods available for the introduction of a sulfonyl fluoride group. Introducing sulfonyl fluorides by way of an aryl boronic acid reagent would also allow combination with the vast number of commercially available aryl halides. In this paper we report the efficient preparation of ortho-, meta-, and parabenzenesulfonyl fluoride boronic acids, together with their reactivity in coupling reactions (Scheme 3).<smiles>[R][R]1ccc(-c2ccc(S(=O)(=O)[O-])cc2)cc1</smiles>

Scheme 3. This work: Benzenesulfonyl fluoride boronic acids and their coupling chemistry.

\section{Results and discussion}

The synthesis of the para-benzenesulfonyl fluoride boronic acid starts from para-bromobenzenesulfonyl chloride, selected for its ready availability and low-cost. Conversion of the sulfonyl chloride group into the corresponding sulfonyl fluoride was achieved by treatment with $\mathrm{KHF}_{2}$ in aqueous acetonitrile at room temperature, ${ }^{3 a}$ and provided sulfonyl fluoride 1a in $98 \%$ yield (Scheme 4a). Miyaura borylation using $\mathrm{B}_{2} \mathrm{pin}_{2}$ with $\mathrm{Pd}(\mathrm{dppf}) \mathrm{Cl}_{2}$ as catalyst delivered boronic ester $2 \mathbf{a}$ in $88 \%$ yield. ${ }^{20}$ Hydrolysis of the boronic ester to liberate the target boronic acid (3a) was achieved using ammonium acetate and sodium periodate in $64 \%$ yield. $^{21}$ para-Benzenesulfonyl fluoride boronic acid 3a was purified by trituration with a hexane/dichloromethane mixture. An identical sequence was completed starting from the meta-sulfonyl chloride, ultimately providing meta-benzenesulfonyl fluoride boronic acid $\mathbf{3 b}$ (Scheme 4b). The ortho-isomer required an alternative route, and the developed synthesis started with commercially available benzenesulfonyl fluoride. Ortho-lithiation of benzenesulfonyl fluoride was achieved using LDA, and in situ quenching with isopropyl borate then provided the corresponding boronic ester, ${ }^{22}$ which was treated directly with aqueous $\mathrm{HCl}$ to provide ortho-benzenesulfonyl fluoride boronic acid (3c) in 59\% yield for this one-pot operation (Scheme 4c).

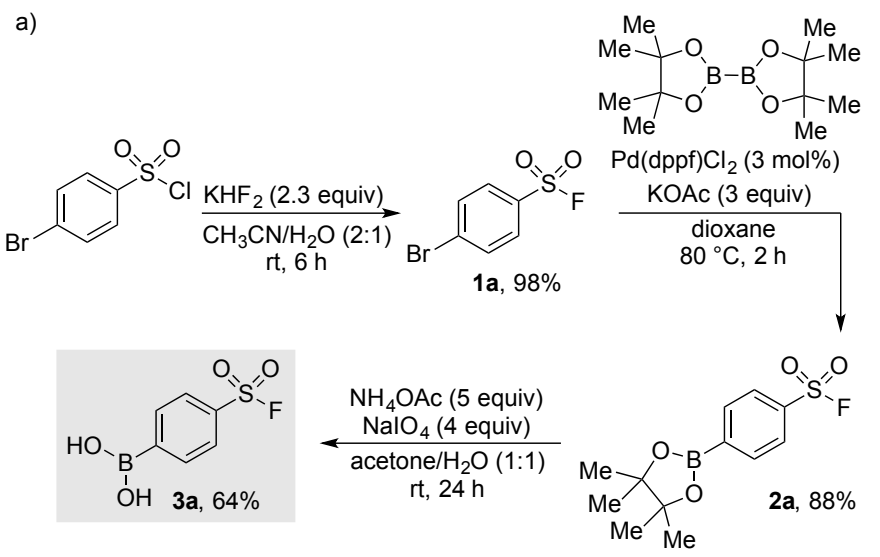

b)

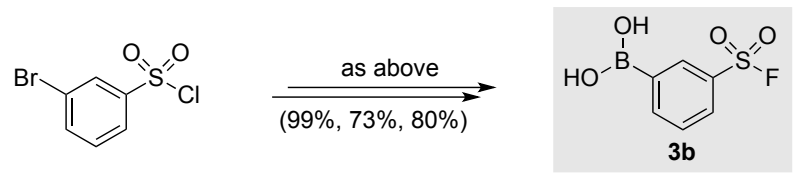

c)

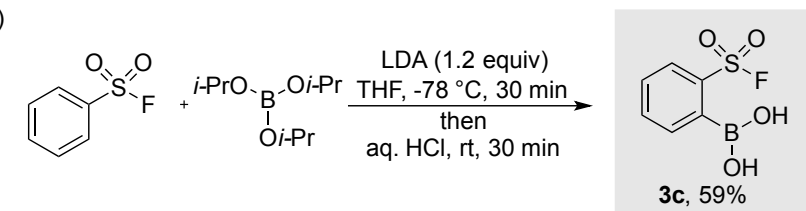

Scheme 4. Preparation of benzenesulfonyl fluoride boronic acids 3a-c.

The routes presented in Scheme 4 employ readily available starting materials and allowed gram-scale quantities of the targeted sulfonyl fluoride boronic acids to be obtained. However, we also wanted to explore a route based on the catalytic sulfination of a substrate featuring an intact boron-substituent. Accordingly, parabromo-substituted aryl pinocolate boronic ester $\mathbf{4}$ was subjected to palladium-catalyzed sulfination conditions, ${ }^{10}$ followed by trapping with the electrophilic fluorinating reagent NFSI, to provide boronic ester substituted sulfonyl fluoride $\mathbf{2 a}$ in $67 \%$ yield. This complementary route to boronic ester $\mathbf{2 a}$ provides an alternative entry to these sulfonyl fluorides, and could find application when late-stage introduction of the sulfur functional group is needed.

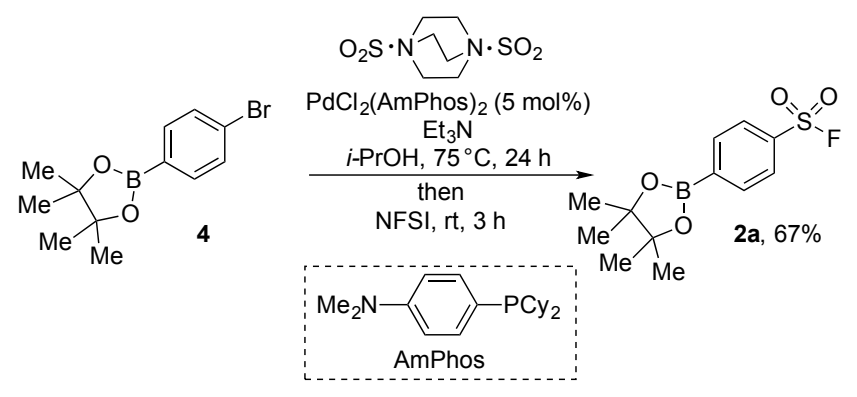

Scheme 5. Sulfonyl fluoride synthesis starting from pinocolate boronic ester $\mathbf{5}$.

With the three key sulfonyl fluoride substituted boronic acids available, we next explored their use in Suzuki-Miyaura coupling 
reactions. We optimized reaction conditions using the coupling between para-substituted boronic acid 3a, and 6-bromoquinoline (Table 1). We evaluated a range of phosphine ligands, using $\mathrm{Pd}(\mathrm{OAc})_{2}$ as the palladium source, and $\mathrm{K}_{3} \mathrm{PO}_{4}$ as base, using dioxane as solvent, initially at $60{ }^{\circ} \mathrm{C}$. Yields were recorded after 2 hours, and 22 hours, with the latter being used to gauge any decomposition of the product under the reaction conditions. Entries 1-7 show that XPhos, SPhos, RuPhos and AmPhos (in this case used as a preformed complex) all performed well under these conditions. XPhos and AmPhos were then evaluated at lower temperatures, with both performing well at $40{ }^{\circ} \mathrm{C}$ (entries 8 and 9). At $25{ }^{\circ} \mathrm{C}$ only XPhos was effective, with the AmPhos system delivering only a low conversion (entries 10 and 11). All entries to this point had used 2.0 equivalents of boronic acid; entry 12 shows that using 1.5 equivalents of boronic acid can achieve good conversion after 4 hours reaction at $40{ }^{\circ} \mathrm{C}$, with minimal decomposition being observed after 22 hours. This final entry represents the conditions that were taken forward.

Table 1. Optimization of the coupling between boronic acid 3a and 6-Br-quinoline. ${ }^{\mathrm{a}}$

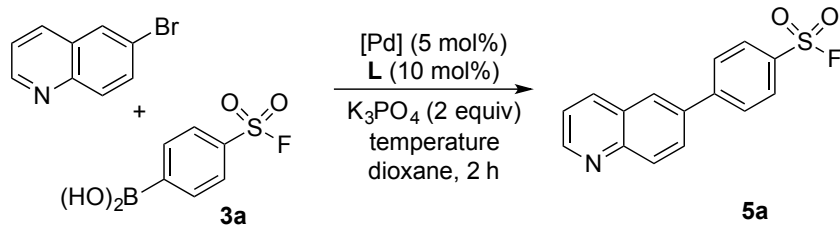

\begin{tabular}{|c|c|c|c|c|c|}
\hline Entry & [Pd] & $\mathbf{L}$ & Temp. & $\begin{array}{c}\text { Yield }^{b} \\
(2 \text { h) }\end{array}$ & $\begin{array}{l}\text { Yield }^{b} \\
(22 \text { h) }\end{array}$ \\
\hline 1 & $\mathrm{Pd}(\mathrm{OAc})_{2}$ & - & $60^{\circ} \mathrm{C}$ & $40 \%$ & $38 \%$ \\
\hline 2 & $\mathrm{Pd}(\mathrm{OAc})_{2}$ & XPhos & $60{ }^{\circ} \mathrm{C}$ & $92 \%$ & $85 \%$ \\
\hline 3 & $\mathrm{Pd}(\mathrm{OAc})_{2}$ & SPhos & $60{ }^{\circ} \mathrm{C}$ & $95 \%$ & $85 \%$ \\
\hline 4 & $\mathrm{Pd}(\mathrm{OAc})_{2}$ & RuPhos & $60^{\circ} \mathrm{C}$ & $96 \%$ & $75 \%$ \\
\hline 5 & $\mathrm{Pd}(\mathrm{OAc})_{2}$ & $\mathrm{PCy}_{3}$ & $60^{\circ} \mathrm{C}$ & $69 \%$ & $60 \%$ \\
\hline 6 & $\mathrm{Pd}\left(\mathrm{PPh}_{3}\right)_{4}$ & - & $60{ }^{\circ} \mathrm{C}$ & $29 \%$ & $53 \%$ \\
\hline 7 & $\mathrm{PdCl}_{2}(\mathrm{AmPhos})_{2}$ & - & $60{ }^{\circ} \mathrm{C}$ & $99 \%$ & $92 \%$ \\
\hline 8 & $\mathrm{Pd}(\mathrm{OAc})_{2}$ & XPhos & $40{ }^{\circ} \mathrm{C}$ & $99 \%$ & $98 \%$ \\
\hline 9 & $\mathrm{PdCl}_{2}(\mathrm{AmPhos})_{2}$ & - & $40^{\circ} \mathrm{C}$ & $99 \%$ & $93 \%$ \\
\hline 10 & $\mathrm{Pd}(\mathrm{OAc})_{2}$ & XPhos & $25^{\circ} \mathrm{C}$ & $81 \%{ }^{\mathrm{c}}$ & $99 \%$ \\
\hline 11 & $\mathrm{PdCl}_{2}(\mathrm{AmPhos})_{2}$ & - & $25^{\circ} \mathrm{C}$ & $10 \%$ & - \\
\hline 12 & $\operatorname{Pd}(\mathrm{OAc})_{2}$ & XPhos & $40^{\circ} \mathrm{C}$ & $94 \%{ }^{d}$ & $93 \%$ \\
\hline
\end{tabular}

a. Reaction conditions: Aryl halide $(0.075 \mathrm{mmol}, 1.0$ equiv), boronic acid (2.0 equiv), [Pd] (5 mol\%), L (10 mol\%), $\mathrm{K}_{3} \mathrm{PO}_{4}(1 \mathrm{M} \mathrm{aq})$ (2.0 equiv), dioxane $[0.25 \mathrm{M}]$, at given temperature. b. Yield determined by HPLC against internal standard. c. 24 hours reaction. d. 4 hours reaction, using 1.5 equiv of boronic acid.

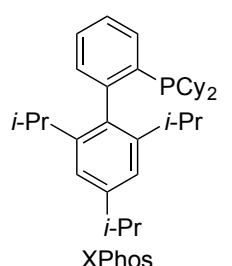<smiles>CCOCCOCCOP(C)c1ccccc1-c1c(OC)cccc1OC</smiles>

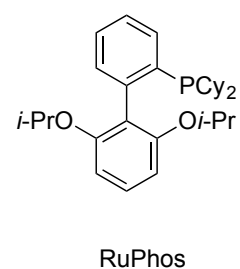

The optimized reaction conditions were then applied to a range of aryl halide coupling partners, initially using para-substituted boronic acid 3a as the fixed component (Table 2). As can be seen, in addition to 6-bromoquinoline (5a), a range of alternative heterocycles could also be successfully employed, including 3bromoquinoline (5b), a 3-bromopyridine (5c), a 3bromothiophene (5d), and a 4-bromopyrazole (5e). Electron-rich and sterically demanding aryl bromides (5f and $\mathbf{5 g}$ ) could be employed, as could a 4-sulfonamide-substituted example (5h). We then employed several more complex aryl halide coupling partners of established worth in medicinal chemistry. For example, the aryl fragments in products $\mathbf{5 i}, \mathbf{5} \mathbf{j}$ and $\mathbf{5 k}$ (a 3-bromopyridine) are present in Sildenafil, Celecoxib, and Omipalisib, ${ }^{23}$ respectively. Meta-sulfonyl fluoride substituted boronic acid $\mathbf{3 b}$ could be used in coupling reactions under identical reaction conditions, and products $\mathbf{5 1}$ and $\mathbf{5 m}$ were obtained in good yields in this way. Product $\mathbf{5 m}$ was obtained using the aryl chloride as the coupling partner, demonstrating that these less reactive aryl halides can be employed providing that an activating substituent, such as trifluoromethyl in this case, is present. The final example in Table 2 shows that the ortho-substituted boronic acid (3c) was significantly more challenging to engage in coupling chemistry than the corresponding para- and meta-isomers. Using the conditions that were successful for the para- and meta-isomers resulted in rapid decomposition of the ortho-sulfonyl fluoride substituted boronic acid. However, the use of KF as base in THF as solvent at room temperature (initial addition at $40{ }^{\circ} \mathrm{C}$ ), ${ }^{24}$ along with an increased amount of palladium catalyst, did restore some reactivity; after 12 hours reaction with 6-bromoquinoline, coupled product 5n was obtained in $43 \%$ yield.

Para-substituted sulfonyl fluoride boronic ester $\mathbf{2 a}$ could also be used as a reaction partner in Suzuki-Miyaura coupling processes, although reduced reactivity, relative to the boronic acid was observed. For example, using the standard conditions, a reaction time of 20 hours was needed to achieve an $80 \%$ yield of biaryl 5a. In contrast, boronic acid 3a delivered coupled product $\mathbf{5 a}$ in $91 \%$ yield after 4 hours reaction. 
Table 2. Suzuki-Miyaura couplings of boronic acids $\mathbf{3 a - c}{ }^{\text {a }}$

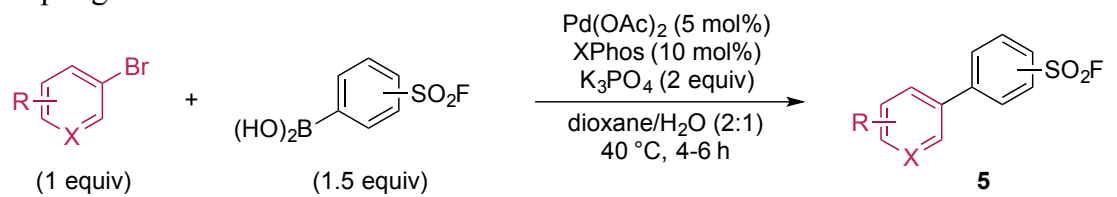

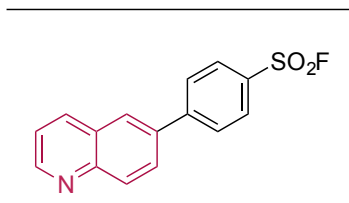

5a, $91 \%$ (80\% using Bpin ester)<smiles>Cn1cc(-c2ccc(S(=O)(=O)F)cc2)cn1</smiles>

$5 e, 43 \%$<smiles>Oc1ccc(-c2cnc3ccccc3c2)cc1</smiles>

5b, $80 \%$<smiles>O=C(c1ccccc1)N1CCN(c2ccc(-c3ccc([N+](=O)[O-])cc3)cn2)CC1</smiles>
$5 c, 80 \%$<smiles>CC(=O)c1sccc1-c1ccc(S(=O)(=O)F)cc1</smiles>
5d, $87 \%$<smiles>O=S(=O)(F)c1ccc(-c2ccc3c(c2)OCO3)cc1</smiles>

5f, $93 \%$<smiles>Cc1cccc(C)c1-c1ccc(S(=O)(=O)F)cc1</smiles>

(17\% from $\mathrm{ArCl}$ )<smiles>NS(=O)(=O)c1ccc(-c2ccc(S(=O)(=O)OF)cc2)cc1</smiles>

5h, $73 \%$<smiles>CCOc1ccc(-c2ccc(S(=O)(=O)F)cc2)cc1-c1nc2c(C(C)C)nn(C)c2c(=O)[nH]1</smiles>

5i, $62 \%$<smiles>Cc1ccc(-c2cc(C(F)(F)F)nn2-c2ccc(-c3ccc(S(=O)(=O)F)cc3)cc2)cc1</smiles>

5j, 95\%<smiles>COc1ncc(-c2ccc(S(=O)(=O)OF)cc2)cc1NS(=O)(=O)c1ccc(F)cc1F</smiles>

$5 k, 42 \%{ }^{b}$

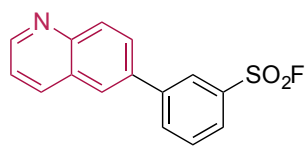

5I, $91 \%$

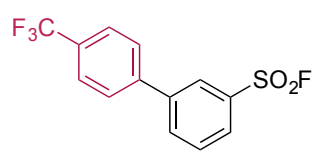

$5 \mathrm{~m}, 64 \%^{\mathrm{c}}$

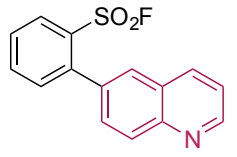

5n, $43 \%^{d}$

a. Reaction conditions: Aryl halide (1.0 equiv), boronic acid (1.5 equiv), $\mathrm{Pd}(\mathrm{OAc}) 2(5 \mathrm{~mol} \%), \mathrm{XPhos}\left(10 \mathrm{~mol}^{\circ}\right), \mathrm{K}_{3} \mathrm{PO} 4(1 \mathrm{M} a q)\left(2.0\right.$ equiv), dioxane, $40{ }^{\circ} \mathrm{C}, 4-6$ h. Isolated yields. b. 24 hours. c. Using aryl chloride substrate. d. Aryl halide (1.0 equiv), boronic acid (1.5 equiv), Pd(OAc) 2 (20 mol\%), XPhos (40 mol\%), KF (3.3 equiv), THF, $40{ }^{\circ} \mathrm{C}, 0.5 \mathrm{~h}$, then $25^{\circ} \mathrm{C}, 12 \mathrm{~h}$.

The rhodium(I)-catalyzed conjugate addition of aryl boronic acids to electron-poor alkenes represents a further useful C-C bond-forming reaction that employs boronic acids, ${ }^{25}$ and accordingly, we were interested in whether sulfonyl fluoride substituted examples would engage in these types of reactions. Scheme 6 shows that para-substituted boronic acid 3a was a competent nucleophile in the addition to cyclohexenone using a simple $\mathrm{Rh}(\mathrm{I})$ catalyst, providing the $\beta$-substituted ketone 6 in $74 \%$ yield.
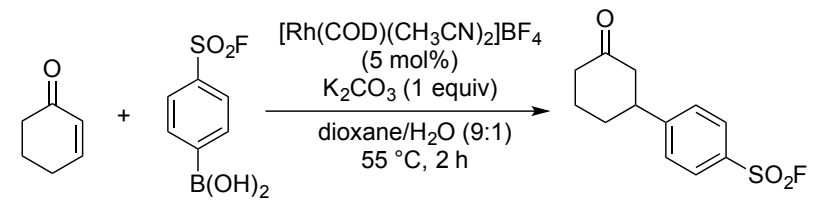

(1 equiv) 3a (2 equiv)

6, $74 \%$

Scheme 6. Rhodium(I)-catalyzed addition of boronic acid 3a to cyclohexenone.

\section{Conclusions}

We have reported efficient and practical syntheses of ortho-, meta, and para-sulfonyl fluoride substituted benzene boronic acids. These boronic acids undergo efficient Sukuki-Miyaura coupling reactions, and we have also achieved an efficient $\mathrm{Rh}(\mathrm{I})$ - catalyzed conjugate addition reaction. These syntheses and reactions provide new methods for the installation of arylsulfonyl fluoride groups and should find applications in medicinal chemistry and chemical biology.

\section{Experimental}

\subsection{General information}

${ }^{1} \mathrm{H},{ }^{11} \mathrm{~B},{ }^{13} \mathrm{C}$, and ${ }^{19} \mathrm{~F}$ NMR spectra were obtained on a Brüker AVIII400 (400 MHz) or AVII (500 MHz) spectrometer. Protondecoupled spectra are denoted as $\left\{{ }^{1} \mathrm{H}\right\}$. Chemical shifts $(\delta)$ were reported in parts per million (ppm) using the residual solvent signal as an internal standard $\left(\mathrm{CDCl}_{3}: \delta_{\mathrm{H}}=7.26 \mathrm{ppm}, \delta_{\mathrm{C}}=77.16\right.$ ppm). All coupling constants ( $J$ values) were reported in Hertz (Hz). Multiplicities were reported as follows: s, singlet; d, doublet; t, triplet; q, quartet; quin., quintet; m, multiplet. High-resolution mass spectrometry (HRMS) measurements were recorded on a Bruker Daltronics microTOF (ESI) spectrometer. Infrared spectra were recorded as thin films on a Bruker Tensor 27 FT-IR spectrometer. Flash chromatography was carried out using matrix 60 silica.

\subsection{Synthesis of bromobenzenesulfonyl fluorides}

\subsubsection{General procedure}


To a solution of $\mathrm{KHF}_{2}$ (2.3 equiv) in deionized water (2 M) was added a solution of bromobenzenesulfonyl chloride ( 1 equiv) in $\mathrm{CH}_{3} \mathrm{CN}(1 \mathrm{M})$. The reaction was stirred at ambient temperature for $24 \mathrm{~h}$. The two phases were separated and the aqueous layer was extracted with EtOAc three times. The combined organic extracts were washed with brine, dried with anhydrous $\mathrm{MgSO}_{4}$, filtered and concentrated in vacuo. The crude product was further purified by flash column chromatography if required to afford the corresponding bromobenzenesulfonyl fluoride.

\subsubsection{4-Bromobenzenesulfonyl fluoride (1a)}

Prepared according to general procedure, using 4bromobenzenesulfonyl chloride $(10.0 \mathrm{~g}, 39.1 \mathrm{mmol}, 1.0$ equiv), $\mathrm{KHF}_{2}$ (7.0 g, $90 \mathrm{mmol}, 2.3$ equiv), deionized water $(20 \mathrm{~mL})$ and $\mathrm{CH}_{3} \mathrm{CN}(40 \mathrm{~mL})$ to afford the corresponding sulfonyl fluoride $\mathbf{1 a}$ as a white solid $(9.16 \mathrm{~g}), 98 \%$ yield. $\boldsymbol{m p} 66-67^{\circ} \mathrm{C}$ (EtOAc); $\mathbf{R}_{\boldsymbol{f}}$ 0.46 (10\% EtOAc in hexane) [UV]; ${ }^{1} \mathbf{H}$ NMR $\left(400 \mathrm{MHz}, \mathrm{CDCl}_{3}\right)$ $\delta 7.88(\mathrm{~d}, J=8.8 \mathrm{~Hz}, 2 \mathrm{H}), 7.79(\mathrm{~d}, J=8.8 \mathrm{~Hz}, 2 \mathrm{H}) ;{ }^{13} \mathbf{C}\left\{{ }^{1} \mathbf{H}\right\} \mathbf{N M R}$ $\left(101 \mathrm{MHz}, \mathrm{CDCl}_{3}\right) \delta 133.3,132.1(\mathrm{~d}, J=25.6 \mathrm{~Hz}), 131.5,130.0$; ${ }^{19} \mathbf{F}\left\{{ }^{1} \mathbf{H}\right\}$ NMR $\left(377 \mathrm{MHz}, \mathrm{CDCl}_{3}\right) \delta 66.4 ; \mathbf{I R} v_{\max }$ (neat) $/ \mathrm{cm}^{-1}$ 3097, 1574, 1406, 1393, 1211, 825, 779, 736; HRMS $\left(\mathrm{EI}^{+}\right) \mathrm{m} / \mathrm{z}$ calc. for $\mathrm{C}_{6} \mathrm{H}_{4}{ }^{79} \mathrm{BrFO}_{2} \mathrm{~S}^{+}[\mathrm{M}]^{+} 237.9094$, found 237.9092 .

\subsubsection{3-Bromobenzenesulfonyl fluoride (1b)}

Prepared according to general procedure, using 3bromobenzenesulfonyl chloride $(2.0 \mathrm{~g}, 7.8 \mathrm{mmol}, 1.0$ equiv), $\mathrm{KHF}_{2}$ (1.4 g, $18 \mathrm{mmol}, 2.3$ equiv), deionized water $(4 \mathrm{~mL})$ and $\mathrm{CH}_{3} \mathrm{CN}(8 \mathrm{~mL})$ to afford the corresponding sulfonyl fluoride $\mathbf{1 b}$ as a pale yellow oil $(1.78 \mathrm{~g}), 99 \%$ yield. $\mathbf{R}_{f} 0.38(10 \%$ EtOAc in hexane) [UV]; ${ }^{1} \mathbf{H}$ NMR $\left(400 \mathrm{MHz}, \mathrm{CDCl}_{3}\right) \delta 8.15(\mathrm{t}, J=1.9 \mathrm{~Hz}$, $1 \mathrm{H}), 7.98-7.94(\mathrm{~m}, 1 \mathrm{H}), 7.93-7.89(\mathrm{~m}, 1 \mathrm{H}), 7.53(\mathrm{td}, J=8.0$, $1.1 \mathrm{~Hz}, 1 \mathrm{H}) ;{ }^{13} \mathbf{C}\left\{{ }^{1} \mathbf{H}\right\}$ NMR $\left(101 \mathrm{MHz}, \mathrm{CDCl}_{3}\right) \delta 138.8,134.8(\mathrm{~d}$, $J=25.8 \mathrm{~Hz}), \quad 131.4, \quad 131.3,127.1, \quad 123.7 ;{ }^{19} \mathbf{F}\left\{{ }^{1} \mathbf{H}\right\} \mathbf{N M R}$ $\left(377 \mathrm{MHz}, \mathrm{CDCl}_{3}\right) \delta 66.3$; IR $v_{\max }$ (neat) $/ \mathrm{cm}^{-1} 3091,1410,1209$, 801, 786, 747; HRMS (EI $) \mathrm{m} / z$ calc. for $\mathrm{C}_{6} \mathrm{H}_{4}{ }^{79} \mathrm{BrFO}_{2} \mathrm{~S}^{+}[\mathrm{M}]^{+}$ 237.9094, found 237.9077 .

\subsection{Synthesis of benzenesulfonyl fluoride boronic acid pinacol esters from bromobenzenesulfonyl fluorides}

\subsubsection{General procedure}

A round bottom flask was charged with 4bromobenzenesulfonyl fluoride $1 \mathrm{a}$ ( 1 equiv), $\mathrm{B}_{2}$ (pin) $)_{2}$ (1.1 equiv), $\mathrm{Pd}(\mathrm{dppf}) \mathrm{Cl}_{2} \cdot \mathrm{CH}_{2} \mathrm{Cl}_{2}(3 \mathrm{~mol} \%)$ and $\mathrm{KOAc}$ (3 equiv), evacuated and back-filled with $\mathrm{N}_{2}$ three times. Degassed anhydrous 1,4dioxane $(0.33 \mathrm{M})$ was added and the reaction was stirred at $80{ }^{\circ} \mathrm{C}$ for $16 \mathrm{~h}$, then cooled to ambient temperature and concentrated in vacuo. The mixture was diluted with EtOAc, washed sequentially with water and brine, dried with anhydrous $\mathrm{MgSO}_{4}$, filtered and concentrated in vacuo. The crude product was purified by flash column chromatography rapidly to afford the corresponding benzenesulfonyl fluoride boronic acid pinacol ester.

\subsubsection{4-(4,4,5,5-Tetramethyl-1,3,2-dioxaborolan-2- yl)benzenesulfonyl fluoride (2a)}

Prepared according to general procedure, using 4bromobenzenesulfonyl fluoride $1 \mathrm{a}$ (4.78 g, $20.0 \mathrm{mmol}, 1.0$ equiv), $\mathrm{B}_{2}$ (pin) $)_{2} \quad\left(5.59 \mathrm{~g}, 22.0 \mathrm{mmol}, 1.1\right.$ equiv), $\mathrm{Pd}(\mathrm{dppf}) \mathrm{Cl}_{2} \cdot \mathrm{CH}_{2} \mathrm{Cl}_{2}$ $(0.49 \mathrm{~g}, 0.60 \mathrm{mmol}, 3 \mathrm{~mol} \%)$, KOAc $(5.88 \mathrm{~g}, 60.0 \mathrm{mmol}$, 3.0 equiv) and 1,4-dioxane $(60 \mathrm{~mL})$. The crude product was purified by flash column chromatography (10-30\% EtOAc in petrol) to afford the corresponding sulfonyl fluoride $\mathbf{2 a}$ as a white solid (5.06 g), 88\% yield. $\boldsymbol{m p} 129-130{ }^{\circ} \mathrm{C}\left(\mathrm{Et}_{2} \mathrm{O}\right) ; \mathbf{R}_{f} 0.23(10 \%$ EtOAc in hexane) $\left[\mathrm{KMnO}_{4} / \mathrm{UV}\right] ;{ }^{1} \mathbf{H}$ NMR $\left(400 \mathrm{MHz}, \mathrm{CDCl}_{3}\right) \delta$ 8.05-8.02 (m, 2H), 8.00-7.96 (m, 2H), $1.36(\mathrm{~s}, 12 \mathrm{H})$; ${ }^{11} \mathbf{B}\left\{{ }^{1} \mathbf{H}\right\}$ NMR $\left(128 \mathrm{MHz}, \mathrm{CDCl}_{3}\right) \quad \delta \quad 30.3 ;{ }^{13} \mathbf{C}\left\{{ }^{1} \mathbf{H}\right\}$ NMR $\left(101 \mathrm{MHz}, \mathrm{CDCl}_{3}\right) \delta 135.8,135.2\left(\mathrm{~d},{ }^{2} J_{\mathrm{CF}}=23.9 \mathrm{~Hz}\right), 127.4,84.9$, 25.0 (1C missing); ${ }^{19} \mathbf{F}\left\{{ }^{1} \mathbf{H}\right\}$ NMR $\left(377 \mathrm{MHz}, \mathrm{CDCl}_{3}\right) \delta 65.7$; IR $v_{\max }($ neat $) / \mathrm{cm}^{-1} 2976,1396,1357,1213,1140,1080,853,768$; HRMS (ESI/APCI/EI) not found.

\subsubsection{3-(4,4,5,5-Tetramethyl-1,3,2-dioxaborolan-2- \\ yl)benzenesulfonyl fluoride (2b)}

Prepared according to general procedure using 3bromobenzenesulfonyl fluoride (1.67 g, $7.0 \mathrm{mmol}, 1.0$ equiv), $\mathrm{B}_{2}$ (pin) $)_{2} \quad\left(1.96 \mathrm{~g}, \quad 7.7 \mathrm{mmol}, 1.1\right.$ equiv), $\mathrm{Pd}(\mathrm{dppf}) \mathrm{Cl}_{2} \cdot \mathrm{CH}_{2} \mathrm{Cl}_{2}$ (172 mg, $0.21 \mathrm{mmol}, 3 \mathrm{~mol} \%)$, KOAc $(2.06 \mathrm{~g}, 21 \mathrm{mmol}$, 3.0 equiv) and 1,4-dioxane $(25 \mathrm{~mL})$. The crude product was purified by flash column chromatography (5-20\% EtOAc in petrol) to afford the corresponding sulfonyl fluoride $\mathbf{2} \mathbf{b}$ as a white solid (1.47 g), $73 \%$ yield. $\boldsymbol{m p} 83-85^{\circ} \mathrm{C}$ (pentane); $\mathbf{R}_{f} 0.21(10 \%$ EtOAc in hexane) $\left[\mathrm{KMnO}_{4} / \mathrm{UV}\right] ;{ }^{1} \mathbf{H}$ NMR $\left(400 \mathrm{MHz}, \mathrm{CDCl}_{3}\right) \delta$ 8.44 (t, $J=1.4 \mathrm{~Hz}, 1 \mathrm{H}), 8.17(\mathrm{~d}, J=7.4 \mathrm{~Hz}, 1 \mathrm{H}), 8.07$ (dt, $J=8.0$, $1.6 \mathrm{~Hz}, 1 \mathrm{H}), 7.62(\mathrm{t}, J=7.7 \mathrm{~Hz}, 1 \mathrm{H}), 1.36(\mathrm{~s}, 12 \mathrm{H}) ;{ }^{11} \mathbf{B}\left\{{ }^{1} \mathbf{H}\right\}$ NMR $\left(128 \mathrm{MHz}, \mathrm{CDCl}_{3}\right) \delta 30.2 ;{ }^{\mathbf{1 3}} \mathbf{C}\left\{{ }^{\mathbf{1}} \mathbf{H}\right\} \mathbf{N M R}\left(101 \mathrm{MHz}, \mathrm{CDCl}_{3}\right) \delta$ 141.7, 134.6, 132.9 (d, $J=23.9 \mathrm{~Hz}), 130.7,129.1,84.9,25.0$ (1C missing); ${ }^{19} \mathbf{F}\left\{{ }^{\mathbf{1}} \mathbf{H}\right\}$ NMR $\left(377 \mathrm{MHz}, \mathrm{CDCl}_{3}\right) \delta$ 65.8; IR $v_{\max }$ (neat) $/ \mathrm{cm}^{-1}$ 2980, 1600, 1406, 1353, 1208, 1142; HRMS (ESI) not found.

\subsection{Pd-catalyzed synthesis of benzenesulfonyl fluoride boronic ester 2 a from bromophenylboronic acid pinacol ester, DABSO and NFSI}

A reaction tube was charged with 4-bromophenylboronic acid pinacol ester ( $85 \mathrm{mg}, 0.30 \mathrm{mmol}, 1.0$ equiv), DABSO (72 mg, $0.30 \mathrm{mmol}, \quad 1.0$ equiv) and $\mathrm{PdCl}_{2}(\text { AmPhos })_{2} \quad$ (10.6 mg, $0.015 \mathrm{mmol}, 5.0 \mathrm{~mol} \%$ ), sealed with a rubber septum, evacuated and back-filled with $\mathrm{N}_{2}$ three times. Anhydrous degassed isopropanol $(1.2 \mathrm{~mL})$ and anhydrous triethylamine $(0.13 \mathrm{~mL}$, $0.90 \mathrm{mmol}, 3.0$ equiv) were added subsequently, and the reaction mixture was stirred under positive pressure of $\mathrm{N}_{2}$ in a preheated aluminium heating block at $75{ }^{\circ} \mathrm{C}$ for $24 \mathrm{~h}$. After cooling to ambient temperature, NFSI (104 mg, $0.33 \mathrm{mmol}, 1.1$ equiv) was added. The reaction mixture was stirred at ambient temperature for $3 \mathrm{~h}$ until completion. The reaction mixture was then diluted with EtOAc, filtered through a plug of Celite ${ }^{\circledR}$ and concentrated in vacuo to yield the crude product which was then purified by flash column chromatography (10-30\% EtOAc in petrol), affording the sulfonyl fluoride $\mathbf{2 a}$ as a white solid $(57 \mathrm{mg}), 67 \%$ yield.

\subsection{Synthesis of arylsulfonyl fluoride boronic acids from the corresponding pinacolate boronic esters}

\subsubsection{General procedure}

4-(Fluorosulfonyl)phenylboronic acid pinacol ester (1 equiv) was dissolved in acetone $(0.2 \mathrm{M})$. A solution of $\mathrm{NH}_{4} \mathrm{OAc}(5$ equiv) in water $(0.2 \mathrm{M})$ was then added, followed by $\mathrm{NaIO}_{4}$ (4 equiv). The mixture was stirred rigorously at ambient temperature for $24 \mathrm{~h}$, volatiles were then removed in vacuo and the residue was extracted with EtOAc. The organic layer was washed with brine, and the combined aqueous extracts were further extracted with EtOAc three times. The combined organic extracts were dried over anhydrous $\mathrm{Na}_{2} \mathrm{SO}_{4}$, filtered and concentrated in vacuo. The 
resultant solid was then triturated with a mixture of hexane/ $\mathrm{CH}_{2} \mathrm{Cl}_{2}$, followed by pentane to afford the corresponding arylsulfonyl fluoride boronic acid.

\subsubsection{4-(Fluorosulfonyl)phenylboronic acid (3a)}

Prepared according to general procedure, using 4(fluorosulfonyl)phenylboronic acid pinacol ester 2a (1.2 g, $4.2 \mathrm{mmol}, 1.0$ equiv), $\mathrm{NH}_{4} \mathrm{OAc}(1.64 \mathrm{~g}, 21 \mathrm{mmol}, 5.0$ equiv), $\mathrm{NaIO}_{4}(3.64 \mathrm{~g}, 17 \mathrm{mmol}, 4.0$ equiv), acetone $(20 \mathrm{~mL})$ and water $(20 \mathrm{~mL})$. The crude product was triturated with hexane $/ \mathrm{CH}_{2} \mathrm{Cl}_{2}$ (2:1), followed by pentane to afford the boronic acid $\mathbf{3 a}$ as a white solid (0.55 g), 64\% yield. $m p 240{ }^{\circ} \mathrm{C}$ (decomp.) $\left(\mathrm{CH}_{2} \mathrm{Cl}_{2}\right) ;{ }^{1} \mathbf{H}$ NMR (400 MHz, DMSO-d $d_{6} \delta 8.58(\mathrm{~s}, 2 \mathrm{H}), 8.14-8.07$ (m, 4H); ${ }^{11} \mathbf{B}\left\{{ }^{1} \mathbf{H}\right\}$ NMR $\left(126 \mathrm{MHz}, \mathrm{DMSO}-d_{6}\right) \delta 27.7 ;{ }^{13} \mathbf{C}\left\{{ }^{1} \mathbf{H}\right\} \mathbf{N M R}$ $\left(101 \mathrm{MHz}, \mathrm{DMSO}-d_{6}\right) \delta 143.9$ (br.), $135.5,132.6$ (d, $J=22.7 \mathrm{~Hz}$ ), $127.0 ;{ }^{19} \mathbf{F}\left\{{ }^{1} \mathbf{H}\right\}$ NMR $\left(377 \mathrm{MHz}, \mathrm{DMSO}-d_{6}\right) \delta 66.1$; IR $v_{\max }$ (neat) $/ \mathrm{cm}^{-1}$ 2160, 1405, 1349, 1304, 1209, 1108, 1075, 1015, 803, 785, 741; LRMS (ESI $\left.{ }^{-}\right) \mathrm{m} / \mathrm{z} 203.0\left([\mathrm{M}-\mathrm{H}]^{-}\right)$; HRMS (ESI $\left.{ }^{-}\right) \mathrm{m} / \mathrm{z}$ calc. for $\mathrm{C}_{6} \mathrm{H}_{5} \mathrm{BFO}_{4} \mathrm{~S}^{-}[\mathrm{M}-\mathrm{H}]^{-} 202.9991$, found 202.9990 .

\subsubsection{3-(Fluorosulfonyl)phenylboronic acid (3b)}

Prepared according to general procedure, using 3(fluorosulfonyl)phenylboronic acid pinacol ester $2 \mathbf{b}$ (1.45 g, $5.1 \mathrm{mmol}, 1.0$ equiv), $\mathrm{NH}_{4} \mathrm{OAc}(1.93 \mathrm{~g}, 25 \mathrm{mmol}, 5.0$ equiv), $\mathrm{NaIO}_{4}(4.28 \mathrm{~g}, 20 \mathrm{mmol}, 4.0$ equiv), acetone $(25 \mathrm{~mL})$ and water $(25 \mathrm{~mL})$. The crude product was triturate with hexane $/ \mathrm{CH}_{2} \mathrm{Cl}_{2}$ (4:1), followed by pentane to afford the boronic acid $\mathbf{3 b}$ as a white solid (0.83 g, 80\%); $\boldsymbol{m p} 240{ }^{\circ} \mathrm{C}$ (decomp.) $\left(\mathrm{CH}_{2} \mathrm{Cl}_{2}\right) ;{ }^{1} \mathbf{H}$ NMR $\left(400 \mathrm{MHz}, \mathrm{DMSO}-d_{6}\right) 8.58$ (s, 2H), 8.46 (app. s, $\left.1 \mathrm{H}\right), 8.29$ (d, $J=$ $7.5 \mathrm{~Hz}, 1 \mathrm{H}), 8.15(\mathrm{ddd}, J=8.0,2.2,1.2 \mathrm{~Hz}, 1 \mathrm{H}), 7.76(\mathrm{t}, J=$ $7.7 \mathrm{~Hz}, 1 \mathrm{H}) ;{ }^{11} \mathbf{B}\left\{{ }^{1} \mathbf{H}\right\}$ NMR $\left(128 \mathrm{MHz}\right.$, DMSO- $\left.d_{6}\right) \delta 27.0$; ${ }^{13} \mathbf{C}\left\{{ }^{1} \mathbf{H}\right\}$ NMR $\left(126 \mathrm{MHz}\right.$, DMSO- $\left.d_{6}\right) \delta 142.0,137.0(\mathrm{~d}, J=$ $20.8 \mathrm{~Hz}), 133.3,131.1(\mathrm{~d}, J=22.3 \mathrm{~Hz}), 129.72,129.70$; ${ }^{19} \mathbf{F}\left\{{ }^{1} \mathbf{H}\right\}$ NMR $\left(377 \mathrm{MHz}, \mathrm{DMSO}-d_{6}\right) \delta 66.4 ; \mathbf{I R} v_{\max }$ (neat) $/ \mathrm{cm}^{-1}$ 1598, 1568, 1401, 1344, 1203, 1091, 841, 762, 721; HRMS (ESI $\left.{ }^{-}\right)$ $m / z$ calc. for $\mathrm{C}_{6} \mathrm{H}_{5} \mathrm{BFO}_{4} \mathrm{~S}^{-}[\mathrm{M}-\mathrm{H}]^{-}$202.9991, found 202.9988.

\subsection{Synthesis of 2-(fluorosulfonyl)phenylboronic acid (3c) through ortho-lithiation/borylation of benzenesulfonyl fluoride}

\section{Preparation of LDA solution}

Diisopropylamine $(0.92 \mathrm{~mL}, \quad 6.6 \mathrm{mmol}, \quad 1.32$ equiv $)$ was dissolved in anhydrous THF $(6 \mathrm{~mL})$ under $\mathrm{N}_{2}$ atmosphere and the solution was cooled to $0{ }^{\circ} \mathrm{C}$. A solution of $n$-BuLi $(2.4 \mathrm{M}$ in hexane) ( $2.5 \mathrm{~mL}, 6.0 \mathrm{mmol}, 1.2$ equiv) was then added dropwise and the mixture was stirred at $0{ }^{\circ} \mathrm{C}$ for $15 \mathrm{~min}$.

\section{Ortho-lithiation/borylation}

Under $\mathrm{N}_{2}$ atmosphere, benzenesulfonyl fluoride $(0.60 \mathrm{~mL}$, $5.0 \mathrm{mmol}, 1.0$ equiv) was dissolved in anhydrous THF $(5 \mathrm{~mL})$ and the solution was cooled to $-78^{\circ} \mathrm{C}$. Triisopropyl borate $(2.8 \mathrm{~mL}$, $12 \mathrm{mmol}, 2.4$ equiv) was added to the solution, followed by the slow addition of LDA solution prepared as described above over $10 \mathrm{~min}$ and the reaction was stirred at $-78{ }^{\circ} \mathrm{C}$ for $30 \mathrm{~min}$. The reaction mixture was then warmed to ambient temperature, $10 \%$ $(\mathrm{v} / \mathrm{v}) a q . \mathrm{HCl}(9 \mathrm{~mL})$ was added and the reaction was stirred at ambient temperature for $30 \mathrm{~min}$. The mixture was then diluted with EtOAc and the two phases were separated. The aqueous extract was further extracted with EtOAc three times and the combined organic extracts were dried over anhydrous $\mathrm{Na}_{2} \mathrm{SO}_{4}$, filtered and concentrated in vacuo. The crude product was purified by flash column chromatography (30-50\% EtOAc in petrol). The resultant oil was added with hexane and sonicated to induce precipitation, which was then subsequently triturated with hexane, hexane $/ \mathrm{CH}_{2} \mathrm{Cl}_{2}(5: 1)$ and pentane to afford the boronic acid $\mathbf{3 c}$ as a white solid (599 mg), 59\% yield. $\boldsymbol{m p} 70-72{ }^{\circ} \mathrm{C}\left(\mathrm{CH}_{2} \mathrm{Cl}_{2}\right) ;{ }^{1} \mathbf{H}$ NMR $\left(400 \mathrm{MHz}, \mathrm{CDCl}_{3}\right) \delta 8.10(\mathrm{~d}, J=8.1 \mathrm{~Hz}, 1 \mathrm{H}), 8.01(\mathrm{~d}, J=$ $7.5 \mathrm{~Hz}, 1 \mathrm{H}), 7.78(\mathrm{t}, J=7.4 \mathrm{~Hz}, 1 \mathrm{H}), 7.67(\mathrm{t}, J=7.4 \mathrm{~Hz}, 1 \mathrm{H}), 5.58$ (br. s, 2H); ${ }^{11} \mathbf{B}\left\{{ }^{1} \mathbf{H}\right\}$ NMR $\quad\left(128 \mathrm{MHz}, \mathrm{CDCl}_{3}\right) \quad \delta \quad 28.7$; ${ }^{13} \mathbf{C}\left\{{ }^{1} \mathbf{H}\right\}$ NMR $\left(101 \mathrm{MHz}, \mathrm{CDCl}_{3}\right) \delta 136.2,135.9(\mathrm{~d}, J=21.6 \mathrm{~Hz})$, 135.0, 131.2 (br.), 130.8, 129.4; ${ }^{\mathbf{1 9}} \mathbf{F}\left\{{ }^{\mathbf{1}} \mathbf{H}\right\}$ NMR $(377 \mathrm{MHz}$, $\left.\mathrm{CDCl}_{3}\right) \delta$ 63.8; IR $v_{\max }$ (neat) $/ \mathrm{cm}^{-1} 3387,1394,1332,1207,790$, 763, 749; HRMS (ESI $\left.{ }^{-}\right) \mathrm{m} / z$ calc. for $\mathrm{C}_{6} \mathrm{H}_{5} \mathrm{BFO}_{4} \mathrm{~S}^{-}[\mathrm{M}-\mathrm{H}]^{-}$ 202.9991, found 202.9991.

\subsection{Suzuki-Miyaura Coupling (SMC) reactions}

\subsubsection{General procedure}

A reaction tube was charged with benzenesulfonyl fluoride boronic acid 3 (61 mg, $0.30 \mathrm{mmol}, 1.5$ equiv), $\mathrm{Pd}(\mathrm{OAc})_{2}(2.3 \mathrm{mg}$, $0.010 \mathrm{mmol}, \quad 5.0 \mathrm{~mol} \%)$ and XPhos (9.5 mg, $0.020 \mathrm{mmol}$, $10 \mathrm{~mol} \%$ ), sealed with a rubber septum, evacuated and back-filled with $\mathrm{N}_{2}$ three times. Degassed 1,4-dioxane $(0.8 \mathrm{~mL})$, degassed $1.0 \mathrm{M}$ aq. $\mathrm{K}_{3} \mathrm{PO}_{4}$ solution ( $0.4 \mathrm{~mL}, 0.40 \mathrm{mmol}, 2.0$ equiv) and aryl halide* $(0.20 \mathrm{mmol}, 1.0$ equiv) were added subsequently, and the reaction mixture stirred under positive pressure of $\mathrm{N}_{2}$ in a preheated aluminium heating block at $40{ }^{\circ} \mathrm{C}$ for $4 \mathrm{~h}(\mathbf{5 a}, \mathbf{b}, \mathbf{f}, \mathbf{g}, \mathbf{h}$, l), $6 \mathrm{~h}(\mathbf{5 c}, \mathbf{d}, \mathbf{e}, \mathbf{i}, \mathbf{j}, \mathbf{m})$ or $24 \mathrm{~h}(\mathbf{5 k})$. After cooling to ambient temperature, the reaction mixture was diluted with EtOAc, dried with anhydrous $\mathrm{MgSO}_{4}$, filtered over Celite ${ }^{\circledR}$ and concentrated in vacuo. The crude product was purified by flash column chromatography to afford the biarylsulfonyl fluoride.

*In the case where aryl halide is a solid, it was added to the vessel before evacuating and back-filling.

\subsubsection{4-(Quinolin-6-yl)benzenesulfonyl fluoride (5a)}

White solid, 91\% yield. $\boldsymbol{m p} 135-136^{\circ} \mathrm{C}\left(\mathrm{CH}_{2} \mathrm{Cl}_{2}\right) ; \mathbf{R}_{\boldsymbol{f}} 0.26$ (30\% EtOAc in hexane) $\left[\mathrm{KMnO}_{4} / \mathrm{UV}\right] ;{ }^{1} \mathbf{H}$ NMR $(400 \mathrm{MHz}$, $\left.\mathrm{CDCl}_{3}\right) \delta 8.99(\mathrm{dd}, J=4.2,1.7 \mathrm{~Hz}, 1 \mathrm{H}), 8.28-8.23(\mathrm{~m}, 2 \mathrm{H}), 8.15-$ $8.12(\mathrm{~m}, 2 \mathrm{H}), 8.07(\mathrm{~d}, J=2.1 \mathrm{~Hz}, 1 \mathrm{H}), 7.99-7.93(\mathrm{~m}, 3 \mathrm{H}), 7.49$ $(\mathrm{dd}, J=8.3,4.2 \mathrm{~Hz}, 1 \mathrm{H}) ;{ }^{13} \mathbf{C}\left\{{ }^{1} \mathbf{H}\right\}$ NMR $\left(101 \mathrm{MHz}, \mathrm{CDCl}_{3}\right) \delta$ 151.6, 148.4, 147.9, 136.7, 136.6, 132.1 (d, $J=24.9 \mathrm{~Hz}), 130.9$, $129.3, \quad 128.7, \quad 128.6, \quad 128.5, \quad 126.9, \quad 122.2 ;{ }^{19} \mathbf{F}\left\{{ }^{1} \mathbf{H}\right\}$ NMR $\left(377 \mathrm{MHz}, \mathrm{CDCl}_{3}\right) \delta 66.5$; IR $v_{\max }$ (neat) $/ \mathrm{cm}^{-1} 3071,1591,1406$, 1212, 1193, 1097; LRMS $\left(\mathrm{ESI}^{+}\right) \mathrm{m} / z 288.0\left([\mathrm{M}+\mathrm{H}]^{+}\right)$; HRMS $\left(\mathrm{ESI}^{+}\right) \mathrm{m} / z$ calc. for $\mathrm{C}_{15} \mathrm{H}_{11} \mathrm{FNO}_{2} \mathrm{~S}^{+}[\mathrm{M}+\mathrm{H}]^{+}$288.0489, found 288.0490 .

Using boronic ester 2a: The general procedure was followed with benzenesulfonyl fluoride boronic ester 2a (86 mg, $0.30 \mathrm{mmol}, 1.5$ equiv) used in place of boronic acid $\mathbf{3 a}$, and the reaction mixture was stirred at $40{ }^{\circ} \mathrm{C}$ for $20 \mathrm{~h}$. Upon purification, biarylsulfonyl fluoride 5a was afforded as a white solid (45.7 mg), $80 \%$ yield. Data as above.

\subsubsection{4-(Quinolin-3-yl)benzenesulfonyl fluoride (5b)}

White solid, $80 \%$ yield. $\boldsymbol{m p} 161-163{ }^{\circ} \mathrm{C}\left(\mathrm{CH}_{2} \mathrm{Cl}_{2}\right) ; \mathbf{R}_{\boldsymbol{f}} 0.42$ (30\% EtOAc in hexane) $\left[\mathrm{KMnO}_{4} / \mathrm{UV}\right] ;{ }^{1} \mathbf{H}$ NMR $(400 \mathrm{MHz}$, $\left.\mathrm{CDCl}_{3}\right) \delta 9.19(\mathrm{~d}, J=2.4 \mathrm{~Hz}, 1 \mathrm{H}), 8.39(\mathrm{dd}, J=2.4,0.8 \mathrm{~Hz}, 1 \mathrm{H})$, 8.21-8.14 (m, 3H), 7.99-7.95 (m, 2H), 7.95-7.92 (m, 1H), 7.81 (ddd, $J=8.4,6.9,1.5 \mathrm{~Hz}, 1 \mathrm{H}), 7.65$ (ddd, $J=8.2,6.9,1.2 \mathrm{~Hz}, 1 \mathrm{H}$ ); ${ }^{13} \mathbf{C}\left\{{ }^{1} \mathbf{H}\right\}$ NMR $\left(101 \mathrm{MHz}, \mathrm{CDCl}_{3}\right) \delta 149.1,148.3,145.5,134.6$, $132.5(\mathrm{~d}, J=25.4 \mathrm{~Hz}), 131.4,130.7,129.6,129.5,128.6,128.4$, 
127.8, 127.8; ${ }^{19} \mathbf{F}\left\{{ }^{1} \mathbf{H}\right\}$ NMR $\left(377 \mathrm{MHz}, \mathrm{CDCl}_{3}\right) \delta 66.5 ; \mathbf{I R} v_{\max }$ (neat) $/ \mathrm{cm}^{-1}$ 3606, 1595, 1403, 1216, 1097; LRMS $\left(\mathrm{ESI}^{+}\right) \mathrm{m} / \mathrm{z}$ $288.0\left([\mathrm{M}+\mathrm{H}]^{+}\right)$; HRMS $\left(\mathrm{ESI}^{+}\right) \mathrm{m} / z$ calc. for $\mathrm{C}_{15} \mathrm{H}_{11} \mathrm{FNO}_{2} \mathrm{~S}^{+}$ $[\mathrm{M}+\mathrm{H}]^{+}$288.0489, found 288.0490.

\subsection{4. tert-Butyl 4-(5-(4-(fluorosulfonyl)phenyl)pyridin-2-} yl)piperazine-1-carboxylate (5c)

White solid, $80 \%$ yield. $\boldsymbol{m p} 198-200{ }^{\circ} \mathrm{C}\left(\mathrm{CHCl}_{3}\right) ; \mathbf{R}_{f} 0.42(30 \%$ EtOAc in hexane) [UV]; ${ }^{1} \mathbf{H}$ NMR $\left(400 \mathrm{MHz}, \mathrm{CDCl}_{3}\right) \delta 8.49$ (dd, $J=2.6,0.7 \mathrm{~Hz}, 1 \mathrm{H}), 8.04(\mathrm{dt}, J=8.5,2.1 \mathrm{~Hz}, 2 \mathrm{H}), 7.77(\mathrm{dd}, J=$ $8.1,1.9 \mathrm{~Hz}, 1 \mathrm{H}), 7.75-7.72(\mathrm{~m}, 2 \mathrm{H}), 6.74(\mathrm{dd}, J=9.0,0.8 \mathrm{~Hz}, 1 \mathrm{H})$, 3.69-3.60 (m, 4H), 3.61-3.50 (m, 4H), 1.49 (s, 9H); ${ }^{13} \mathbf{C}\left\{{ }^{1} \mathbf{H}\right\}$ NMR $\left(101 \mathrm{MHz}, \mathrm{CDCl}_{3}\right) \delta 159.3,154.9,147.0,145.9$, $136.3,130.6(\mathrm{~d}, J=24.8 \mathrm{~Hz}), 129.3,126.8,123.4,107.0,80.3$, 44.9, 43.3 (br.), 28.6; ${ }^{19} \mathbf{F}\left\{{ }^{1} \mathbf{H}\right\}$ NMR $\left(377 \mathrm{MHz}, \mathrm{CDCl}_{3}\right) \delta 66.6$; IR $v_{\max }$ (neat) $/ \mathrm{cm}^{-1} 2981,2923,1673,1605,1589,1424,1403$, 1243, 1212, 1190, 1172; LRMS $\left(\mathrm{ESI}^{+}\right) \mathrm{m} / \mathrm{z} 422.0\left([\mathrm{M}+\mathrm{H}]^{+}\right)$; HRMS $\left(\mathrm{ESI}^{+}\right) \mathrm{m} / z$ calc. for $\mathrm{C}_{20} \mathrm{H}_{25} \mathrm{FN}_{3} \mathrm{O}_{4} \mathrm{~S}^{+}[\mathrm{M}+\mathrm{H}]^{+} 422.1544$, found 422.1548 .

\subsubsection{4-(2-Acetylthiophen-3-yl)benzenesulfonyl fluoride (5d)}

Off-white solid, $87 \%$ yield. $\boldsymbol{m p} 127-129{ }^{\circ} \mathrm{C}\left(\mathrm{CHCl}_{3}\right) ; \mathbf{R}_{\boldsymbol{f}} 0.44$ (30\% EtOAc in hexane) [UV]; ${ }^{1} \mathbf{H}$ NMR $\left(400 \mathrm{MHz}, \mathrm{CDCl}_{3}\right) \delta 8.05$ $(\mathrm{dt}, J=8.5,1.8 \mathrm{~Hz}, 2 \mathrm{H}), 7.66(\mathrm{dtd}, J=8.8,1.9,0.8 \mathrm{~Hz}, 2 \mathrm{H}), 7.61$ $(\mathrm{d}, J=5.1 \mathrm{~Hz}, 1 \mathrm{H}), 7.09(\mathrm{~d}, J=5.0 \mathrm{~Hz}, 1 \mathrm{H}), 2.39(\mathrm{~s}, 3 \mathrm{H})$; ${ }^{13} \mathbf{C}\left\{{ }^{1} \mathbf{H}\right\}$ NMR $\left(101 \mathrm{MHz}, \mathrm{CDCl}_{3}\right) \delta 190.5,144.3,144.1,138.9$, $132.6(\mathrm{~d}, J=25.3 \mathrm{~Hz}), 131.9,131.2,130.7,128.5,29.9$; ${ }^{19} \mathbf{F}\left\{{ }^{1} \mathbf{H}\right\}$ NMR $\left(377 \mathrm{MHz}, \mathrm{CDCl}_{3}\right) \delta 66.2 ; \mathbf{I R} v_{\max }$ (neat) $/ \mathrm{cm}^{-1}$ 3105, 1672, 1402, 1274, 1209, 1186, 1095; LRMS $\left(\mathrm{ESI}^{+}\right) \mathrm{m} / \mathrm{z}$ $285.0\left([\mathrm{M}+\mathrm{H}]^{+}\right)$; HRMS $\left(\mathrm{ESI}^{+}\right) \mathrm{m} / \mathrm{z}$ calc. for $\mathrm{C}_{12} \mathrm{H}_{10} \mathrm{FO}_{3} \mathrm{~S}_{2}{ }^{+}$ $[\mathrm{M}+\mathrm{H}]^{+} 285.0050$, found 285.0051 .

4.7.6. 4-(1-Methyl-1H-pyrazol-4-yl)benzenesulfonyl fluoride (5e)

White solid, $43 \%$ yield. $\boldsymbol{m p} 85-86^{\circ} \mathrm{C}\left(\mathrm{CHCl}_{3}\right) ; \mathbf{R}_{f} 0.29(50 \%$ EtOAc in hexane) [UV]; ${ }^{1} \mathbf{H}$ NMR $\left(400 \mathrm{MHz}, \mathrm{CDCl}_{3}\right) \delta 7.98$ (dt, $J=8.5,1.9 \mathrm{~Hz}, 2 \mathrm{H}), 7.85(\mathrm{~d}, J=0.9 \mathrm{~Hz}, 1 \mathrm{H}), 7.75(\mathrm{~d}, J=0.8 \mathrm{~Hz}$, 1H), 7.67 (dtd, $J=8.8,2.1,1.0 \mathrm{~Hz}, 2 \mathrm{H}), 3.99$ (s, 3H); ${ }^{13} \mathbf{C}\left\{{ }^{1} \mathbf{H}\right\}$ NMR $\left(101 \mathrm{MHz}, \mathrm{CDCl}_{3}\right) \delta 140.5,137.5,130.0(\mathrm{~d}, J=$ $24.3 \mathrm{~Hz}), \quad 129.4, \quad 128.3 ， 126.0,121.1 ， 39.5 ;{ }^{19} \mathbf{F}\left\{{ }^{1} \mathbf{H}\right\} \mathbf{~ N M R}$ $\left(377 \mathrm{MHz}, \mathrm{CDCl}_{3}\right) \delta 66.5 ;$ IR $v_{\max }$ (neat) $/ \mathrm{cm}^{-1} 3101,2948,1597$, 1564, 1402, 1206, 1192, 1097; LRMS $\left(\mathrm{ESI}^{+}\right) \mathrm{m} / z 241.0\left([\mathrm{M}+\mathrm{H}]^{+}\right)$; HRMS $\left(\mathrm{ESI}^{+}\right) \mathrm{m} / z$ calc. for $\mathrm{C}_{10} \mathrm{H}_{10} \mathrm{FN}_{2} \mathrm{O}_{2} \mathrm{~S}^{+}[\mathrm{M}+\mathrm{H}]^{+}$241.0442, found 241.0443 .

\subsubsection{4-(Benzo[d][1,3]dioxol-5-yl)benzenesulfonyl fluoride (5f)}

White solid, $93 \%$ yield. $\boldsymbol{m p} 104-105{ }^{\circ} \mathrm{C}\left(\mathrm{CHCl}_{3}\right) ; \mathbf{R}_{f} 0.45(20 \%$ EtOAc in hexane) [UV]; ${ }^{1} \mathbf{H}$ NMR $\left(400 \mathrm{MHz}, \mathrm{CDCl}_{3}\right) \delta 8.03$ (dtd, $J=8.8,2.0,0.5 \mathrm{~Hz}, 2 \mathrm{H}), 7.74(\mathrm{dtd}, J=8.8,2.1,0.9 \mathrm{~Hz}, 2 \mathrm{H}), 7.12$ $(\mathrm{dd}, J=8.0,1.9 \mathrm{~Hz}, 1 \mathrm{H}), 7.09(\mathrm{dd}, J=1.9,0.4 \mathrm{~Hz}, 1 \mathrm{H}), 6.93(\mathrm{~d}, J$ $=8.0 \mathrm{~Hz}, 1 \mathrm{H}), 6.05(\mathrm{~s}, 2 \mathrm{H}) ;{ }^{13} \mathbf{C}\left\{{ }^{1} \mathbf{H}\right\} \mathbf{N M R}\left(101 \mathrm{MHz}, \mathrm{CDCl}_{3}\right) \delta$ $148.9,148.8,148.4,132.8,131.0(\mathrm{~d}, J=24.6 \mathrm{~Hz}), 129.1,127.9$, 121.7, 109.1, 107.8, 101.8; ${ }^{19} \mathbf{F}\left\{{ }^{1} \mathbf{H}\right\}$ NMR $\left(377 \mathrm{MHz}, \mathrm{CDCl}_{3}\right) \delta$ 66.5; IR $v_{\max }$ (neat) $/ \mathrm{cm}^{-1} 3079,2922,1594,1505,1477,1442$, 1405, 1223, 1209, 1186, 1100, 1033; HRMS (ESI) not found.

\subsubsection{2',6'-Dimethyl-[1,1'-biphenyl]-4-sulfonyl fluoride (5g)}

White solid, $67 \%$ yield. $\boldsymbol{m p} 133-134{ }^{\circ} \mathrm{C}\left(\mathrm{CHCl}_{3}\right) ; \mathbf{R}_{f} 0.56(10 \%$ EtOAc in hexane) [UV]; ${ }^{1} \mathbf{H}$ NMR $\left(400 \mathrm{MHz}, \mathrm{CDCl}_{3}\right) \delta 8.09$ (dtd, $J=8.2,2.0,0.4 \mathrm{~Hz}, 2 \mathrm{H}), 7.44(\mathrm{dtd}, J=8.6,1.9,0.9 \mathrm{~Hz}, 2 \mathrm{H}), 7.23$ $(\mathrm{dd}, J=8.4,6.7 \mathrm{~Hz}, 1 \mathrm{H}), 7.16-7.13(\mathrm{~m}, 2 \mathrm{H}), 2.01(\mathrm{~s}, 6 \mathrm{H})$;
${ }^{13} \mathbf{C}\left\{{ }^{1} \mathbf{H}\right\}$ NMR $\left(101 \mathrm{MHz}, \mathrm{CDCl}_{3}\right) \delta 149.6,139.4,135.4,131.6$ $(\mathrm{d}, J=24.6 \mathrm{~Hz}), 130.8,128.9,128.3,127.9,20.9 ;{ }^{19} \mathbf{F}\left\{{ }^{1} \mathbf{H}\right\}$ NMR $\left(377 \mathrm{MHz}, \mathrm{CDCl}_{3}\right) \delta 66.1$; IR $v_{\max }$ (neat) $/ \mathrm{cm}^{-1} 3088,2966,2923$, 1590, 1464, 1403, 1208, 1179, 1163, 1092; HRMS (ESI) not found.

\subsubsection{4'-Sulfamoyl-[1,1'-biphenyl]-4-sulfonyl fluoride (5h)}

White solid, 73\% yield. $\boldsymbol{m p} 181-183{ }^{\circ} \mathrm{C}$ (EtOAc); $\mathbf{R}_{\boldsymbol{f}} 0.14(30 \%$ EtOAc in hexane) [UV]; ${ }^{1} \mathbf{H}$ NMR (400 MHz, DMSO- $\left.d_{6}\right) \delta 8.25$ $(\mathrm{d}, J=8.6 \mathrm{~Hz}, 2 \mathrm{H}), 8.14(\mathrm{~d}, J=8.1 \mathrm{~Hz}, 2 \mathrm{H}), 8.02-7.95(\mathrm{~m}, 4 \mathrm{H})$, $7.49(\mathrm{~s}, 2 \mathrm{H}) ;{ }^{13} \mathbf{C}\left\{{ }^{1} \mathbf{H}\right\}$ NMR $\left(101 \mathrm{MHz}, \mathrm{CDCl}_{3}\right) \delta$ 146.4, 144.6, $140.7,130.9(\mathrm{~d}, J=23.9 \mathrm{~Hz}), 129.2,128.9,128.1,126.5$; ${ }^{19} \mathbf{F}\left\{{ }^{1} \mathbf{H}\right\}$ NMR $\left(377 \mathrm{MHz}, \mathrm{CDCl}_{3}\right) \delta 66.6$; IR $v_{\max }$ (neat) $/ \mathrm{cm}^{-1}$ 3367, 3263, 1594, 1404, 1329, 1215, 1153, 1097; LRMS (ESI $\left.{ }^{-}\right)$ $m / z 313.9\left([\mathrm{M}-\mathrm{H}]^{-}\right)$; HRMS $\left(\mathrm{ESI}^{-}\right) \mathrm{m} / z$ calc. for $\mathrm{C}_{12} \mathrm{H}_{9} \mathrm{FNO}_{4} \mathrm{~S}_{2}{ }^{-}$ $[\mathrm{M}-\mathrm{H}]^{-}$313.9963, found 313.9961.

\subsubsection{4'-Ethoxy-3'-(1-methyl-7-oxo-3-propyl-6,7-dihydro-1H- pyrazolo[4,3-d]pyrimidin-5-yl)-[1,1'-biphenyl]-4-sulfonyl fluoride (5i)}

5-(5-Bromo-2-ethoxyphenyl)-1-methyl-3-propyl-1,6-dihydro7H-pyrazolo[4,3- $d]$ pyrimidin-7-one $\quad(39.1 \mathrm{mg}, \quad 0.10 \mathrm{mmol}$, 1.0 equiv), 4-(fluorosulfonyl)phenylboronic acid $\mathbf{3 a}(31 \mathrm{mg}$, $0.15 \mathrm{mmol}, 1.5$ equiv), $\mathrm{Pd}(\mathrm{OAc})_{2}(1.1 \mathrm{mg}, 5.0 \mu \mathrm{mol}, 5.0 \mathrm{~mol} \%)$, XPhos (4.8 mg, $10 \mu \mathrm{mol}, 10 \mathrm{~mol} \%), 1.0 \mathrm{M}$ aq. $\mathrm{K}_{3} \mathrm{PO}_{4}$ solution $(0.2 \mathrm{~mL}, 0.20 \mathrm{mmol}, 2.0$ equiv) and 1,4-dioxane $(0.4 \mathrm{~mL})$ were used. Sulfonyl fluoride $\mathbf{5 i}$ was isolated as a white solid $(29.0 \mathrm{mg})$, $62 \%$ yield. $\boldsymbol{m p} 176-178{ }^{\circ} \mathrm{C}\left(\mathrm{CHCl}_{3}\right) ; \mathbf{R}_{\boldsymbol{f}} 0.26(50 \%$ EtOAc in hexane) $\left[\mathrm{KMnO}_{4} / \mathrm{UV}\right] ;{ }^{1} \mathbf{H}$ NMR $\left(400 \mathrm{MHz}, \mathrm{CDCl}_{3}\right) \delta 10.94(\mathrm{~s}$, $1 \mathrm{H}), 8.67$ (d, $J=2.5 \mathrm{~Hz}, 1 \mathrm{H}), 8.08-8.00(\mathrm{~m}, 2 \mathrm{H}), 7.80$ (dd, $J=8.9$, $0.8 \mathrm{~Hz}, 2 \mathrm{H}), 7.65(\mathrm{dd}, J=8.6,2.5 \mathrm{~Hz}, 1 \mathrm{H}), 7.11(\mathrm{~d}, J=8.7 \mathrm{~Hz}$, $1 \mathrm{H}), 4.35-4.26(\mathrm{~m}, 2 \mathrm{H}), 4.21(\mathrm{~s}, 3 \mathrm{H}), 2.87(\mathrm{dd}, J=8.0,7.1 \mathrm{~Hz}$, 2H), 1.80 (h, $J=7.4 \mathrm{~Hz}, 2 \mathrm{H}), 1.58(\mathrm{t}, J=7.0 \mathrm{~Hz}, 3 \mathrm{H}), 0.96$ (t, $J=$ $7.4 \mathrm{~Hz}, 3 \mathrm{H})$ (recorded as a mixture of tautomers at ratio of 82:13:5); ${ }^{13} \mathbf{C}\left\{{ }^{1} \mathbf{H}\right\}$ NMR $\left(101 \mathrm{MHz}, \mathrm{CDCl}_{3}\right) \delta 157.3,153.9$, $147.8,147.4,146.9,138.6,132.2,131.5$ (d, $J=24.7 \mathrm{~Hz}), 131.2$, $130.3,129.3,128.0,124.7,121.2,113.9,65.9,38.4,27.9,22.5$, $14.8,14.2 ;{ }^{19} \mathbf{F}\left\{{ }^{1} \mathbf{H}\right\}$ NMR $\left(377 \mathrm{MHz}, \mathrm{CDCl}_{3}\right) \delta 66.5 ; \mathbf{I R} v_{\max }$ (neat) $/ \mathrm{cm}^{-1} 3310,2959,2932,1697,1593,1566,1486,1473,1406$, 1213, 1159, 1031; LRMS (ESI $\left.{ }^{+}\right) m / z 471.2\left([\mathrm{M}+\mathrm{H}]^{+}\right)$; HRMS $\left(\mathrm{ESI}^{+}\right) \mathrm{m} / z$ calc. for $\mathrm{C}_{23} \mathrm{H}_{24} \mathrm{FN}_{4} \mathrm{O}_{4} \mathrm{~S}^{+}[\mathrm{M}+\mathrm{H}]^{+} 471.1497$, found 471.1496.

4.7.11. 4'-(5-(p-Tolyl)-3-(trifluoromethyl)-1H-pyrazol-1-yl)-[1,1'biphenyl]-4-sulfonyl fluoride (5j)

1-(4-Bromophenyl)-5-(p-tolyl)-3-(trifluoromethyl)-1H-

pyrazole $\quad(38.1 \mathrm{mg}, \quad 0.10 \mathrm{mmol}, \quad 1.0$ equiv), 4-(fluorosulfonyl)phenylboronic acid $3 a$ (31 mg, $0.15 \mathrm{mmol}$, 1.5 equiv $), \operatorname{Pd}(\mathrm{OAc})_{2}(1.1 \mathrm{mg}, 5.0 \mu \mathrm{mol}, 5.0 \mathrm{~mol} \%)$, XPhos $(4.8 \mathrm{mg}, 10 \mu \mathrm{mol}, 10 \mathrm{~mol} \%), 1.0 \mathrm{M}$ aq. $\mathrm{K}_{3} \mathrm{PO}_{4}$ solution $(0.2 \mathrm{~mL}$, $0.20 \mathrm{mmol}, 2.0$ equiv) and 1,4-dioxane $(0.4 \mathrm{~mL})$ were used. Sulfonyl fluoride $\mathbf{5} \mathbf{j}$ was isolated as an off-white solid (43.5 $\mathrm{mg}$ ), $95 \%$ yield. $\boldsymbol{m p} 121-123{ }^{\circ} \mathrm{C}\left(\mathrm{CHCl}_{3}\right) ; \mathbf{R}_{\boldsymbol{f}} 0.41(10 \%$ EtOAc in hexane) [UV]; ${ }^{1} \mathbf{H}$ NMR (400 MHz, $\left.\mathrm{CDCl}_{3}\right) \delta 8.09$ (ddt, $J=103.1$, $8.5,2.0 \mathrm{~Hz}, 2 \mathrm{H}), 7.82(\mathrm{dtd}, J=8.9,2.1,0.8 \mathrm{~Hz}, 2 \mathrm{H}), 7.62(\mathrm{dt}, J=$ 8.7, 2.5 Hz, 2H), 7.47 (dt, $J=8.8,2.2 \mathrm{~Hz}, 2 \mathrm{H}), 7.19-7.15(\mathrm{~m}, 4 \mathrm{H})$, $6.75(\mathrm{~s}, 1 \mathrm{H}), 2.38(\mathrm{~s}, 3 \mathrm{H}) ;{ }^{13} \mathbf{C}\left\{{ }^{1} \mathbf{H}\right\}$ NMR $\left(101 \mathrm{MHz}, \mathrm{CDCl}_{3}\right) \delta$ 147.3, 145.1, 143.8 (d, $J=38.9 \mathrm{~Hz}), 140.2,139.6,138.4,132.1$ (d, $J=24.7 \mathrm{~Hz}), 129.7,129.2,128.9,128.3,128.2,126.3,126.1$, $121.4(\mathrm{~d}, J=269.5 \mathrm{~Hz}), 106.0,21.5 ;{ }^{19} \mathbf{F}$ NMR $\left(377 \mathrm{MHz}, \mathrm{CDCl}_{3}\right)$ $\delta 66.5(\mathrm{~s}, 1 \mathrm{~F}),-62.3(\mathrm{~s}, 3 \mathrm{~F})$; IR $v_{\max }$ (neat) $/ \mathrm{cm}^{-1} 3061,2925,1595$, 1472, 1408, 1376, 1236, 1213, 1161, 1133, 1097; LRMS $\left(\mathrm{ESI}^{+}\right)$ 
$m / z \quad 461.0 \quad\left([\mathrm{M}+\mathrm{H}]^{+}\right) ; \quad$ HRMS $\quad\left(\mathrm{ESI}^{+}\right) \quad \mathrm{m} / \mathrm{z}$ calc. for $\mathrm{C}_{21} \mathrm{H}_{17} \mathrm{~F}_{4} \mathrm{~N}_{2} \mathrm{O}_{2} \mathrm{~S}^{+}[\mathrm{M}+\mathrm{H}]^{+}$461.0941, found 461.0938.

\subsubsection{4-(5-((2,4-Difluorophenyl) sulfonamido)-6- methoxypyridin-3-yl)benzenesulfonyl fluoride (5k)}

$\mathrm{N}$-(5-Bromo-2-methoxypyridin-3-yl)-2,4-difluorobenzenesulfonamide $\quad(37.9 \mathrm{mg}, \quad 0.10 \mathrm{mmol}, \quad 1.0$ equiv), 4-(fluorosulfonyl)phenylboronic acid $3 \mathbf{a}$ (31 $\mathrm{mg}, 0.15 \mathrm{mmol}$, 1.5 equiv), $\mathrm{Pd}(\mathrm{OAc})_{2}(1.1 \mathrm{mg}, 5.0 \mu \mathrm{mol}, 5.0 \mathrm{~mol} \%)$, XPhos $(4.8 \mathrm{mg}, 10 \mu \mathrm{mol}, 10 \mathrm{~mol} \%), 1.0 \mathrm{M}$ aq. $\mathrm{K}_{3} \mathrm{PO}_{4}$ solution $(0.2 \mathrm{~mL}$, $0.20 \mathrm{mmol}, 2.0$ equiv) and 1,4-dioxane $(0.4 \mathrm{~mL})$ were used. Sulfonyl fluoride $\mathbf{5 k}$ was isolated as an off-white solid (19.4 mg), $42 \%$ yield. $\boldsymbol{m} \boldsymbol{p} 152-154{ }^{\circ} \mathrm{C}\left(\mathrm{CHCl}_{3}\right) ; \mathbf{R}_{\boldsymbol{f}} 0.52$ (50\% EtOAc in hexane) [UV]; ${ }^{1} \mathbf{H}$ NMR $\left(500 \mathrm{MHz}, \mathrm{CDCl}_{3}\right) \delta 8.13(\mathrm{~d}, J=2.3 \mathrm{~Hz}$, $1 \mathrm{H}), 8.08$ (dt, $J=8.6,2.0 \mathrm{~Hz}, 2 \mathrm{H}), 7.99$ (d, $J=2.3 \mathrm{~Hz}, 1 \mathrm{H}), 7.91-$ $7.86(\mathrm{~m}, 1 \mathrm{H}), 7.72(\mathrm{~d}, J=8.4 \mathrm{~Hz}, 2 \mathrm{H}), 7.28$ (br. s, $1 \mathrm{H}), 6.98-6.93$ $(\mathrm{m}, 2 \mathrm{H}), 3.98(\mathrm{~s}, 3 \mathrm{H}) ;{ }^{13} \mathbf{C}\left\{{ }^{1} \mathbf{H},{ }^{19} \mathbf{F}\right\}$ NMR $\left(126 \mathrm{MHz}, \mathrm{CDCl}_{3}\right) \delta$ $166.4,160.0,155.0,144.7,140.9,132.5,129.4,128.5,127.9$, 126.3, 123.5, 121.0, 112.2, 106.0, 54.6 (1C missing); ${ }^{19} \mathbf{F}$ NMR $\left(377 \mathrm{MHz}, \mathrm{CDCl}_{3}\right) \delta 66.4(\mathrm{~s}, 1 \mathrm{~F}),-98.5(\mathrm{~m}, 1 \mathrm{~F}),-103.8(\mathrm{~m}, 1 \mathrm{~F})$; IR $v_{\max }$ (neat) $/ \mathrm{cm}^{-1} 3285,3103,2929,2856,1602,1480,1407$, 1347, 1213, 1178, 1149; LRMS $\left(\mathrm{ESI}^{+}\right) \mathrm{m} / \mathrm{z} 459.0\left([\mathrm{M}+\mathrm{H}]^{+}\right)$; HRMS $\left(\mathrm{ESI}^{+}\right) \mathrm{m} / z$ calc. for $\mathrm{C}_{18} \mathrm{H}_{14} \mathrm{~F}_{3} \mathrm{~N}_{2} \mathrm{O}_{5} \mathrm{~S}_{2}{ }^{+}[\mathrm{M}+\mathrm{H}]^{+} 459.0291$, found 459.0291 .

\subsubsection{3-(Quinolin-6-yl)benzenesulfonyl fluoride (5l)}

White solid, 91\% yield. $\boldsymbol{m p} 111-113{ }^{\circ} \mathrm{C}\left(\mathrm{CHCl}_{3}\right) ; \mathbf{R}_{f} 0.24(30 \%$ EtOAc in hexane) [UV]; ${ }^{1} \mathbf{H}$ NMR $\left(400 \mathrm{MHz}, \mathrm{CDCl}_{3}\right) \delta 8.98(\mathrm{dd}$, $J=4.2,1.7 \mathrm{~Hz}, 1 \mathrm{H}), 8.34(\mathrm{t}, J=1.9 \mathrm{~Hz}, 1 \mathrm{H}), 8.27-8.22(\mathrm{~m}, 2 \mathrm{H})$, 8.12-8.08 (m, 1H), 8.06-8.02 (m, 2H), $7.96(\mathrm{dd}, J=8.8,2.2 \mathrm{~Hz}$, $1 \mathrm{H}), 7.77(\mathrm{t}, J=7.9 \mathrm{~Hz}, 1 \mathrm{H}), 7.48(\mathrm{dd}, J=8.3,4.2 \mathrm{~Hz}, 1 \mathrm{H})$; ${ }^{13} \mathbf{C}\left\{{ }^{1} \mathbf{H}\right\}$ NMR $\left(101 \mathrm{MHz}, \mathrm{CDCl}_{3}\right) \delta 151.4,148.2,142.5,136.5$, $136.4,134.4,134.1$ (d, $J=24.6 \mathrm{~Hz}), 130.8,130.5,128.5(2 \mathrm{C})$, $127.4,127.2,126.5,122.1 ;{ }^{19} \mathbf{F}\left\{{ }^{1} \mathbf{H}\right\}$ NMR $\left(377 \mathrm{MHz}, \mathrm{CDCl}_{3}\right) \delta$ 66.1; IR $v_{\max }\left(\right.$ neat) $/ \mathrm{cm}^{-1} 3067,1593,1480,1404,1208$; LRMS $\left(\mathrm{ESI}^{+}\right) \mathrm{m} / \mathrm{z} 288.0\left([\mathrm{M}+\mathrm{H}]^{+}\right)$; HRMS $\left(\mathrm{ESI}^{+}\right) \mathrm{m} / \mathrm{z}$ calc. for $\mathrm{C}_{15} \mathrm{H}_{11} \mathrm{FNO}_{2} \mathrm{~S}^{+}[\mathrm{M}+\mathrm{H}]^{+}$288.0489, found 288.0489.

\subsubsection{4'-(Trifluoromethyl)-[1,1'-biphenyl]-3-sulfonyl fluoride (5m)}

Off-white solid, $64 \%$ yield. $\boldsymbol{m p} 50-51{ }^{\circ} \mathrm{C}\left(\mathrm{CHCl}_{3}\right) ; \mathbf{R}_{f} 0.70$ $\left(30 \%\right.$ EtOAc in hexane) $\left[\mathrm{UV} / \mathrm{KMnO}_{4}\right] ;{ }^{1} \mathbf{H}$ NMR $(500 \mathrm{MHz}$, $\left.\mathrm{CDCl}_{3}\right) \delta 8.22(\mathrm{t}, J=1.9 \mathrm{~Hz}, 1 \mathrm{H}), 8.06(\mathrm{dt}, J=7.9,1.5 \mathrm{~Hz}, 1 \mathrm{H})$, $8.00(\mathrm{dt}, J=7.8,1.4 \mathrm{~Hz}, 1 \mathrm{H}), 7.78-7.71(\mathrm{~m}, 5 \mathrm{H}) ;{ }^{13} \mathbf{C}\left\{{ }^{\mathbf{1}} \mathbf{H}\right\} \mathbf{N M R}$ $\left(101 \mathrm{MHz}, \mathrm{CDCl}_{3}\right) \delta 141.9,141.9,134.3,134.2(\mathrm{~d}, J=24.6 \mathrm{~Hz})$, 131.1 (app. d, $J=32.7 \mathrm{~Hz}$ ), 130.6, 127.9, 127.8, 127.2, 126.4 (q, $J$ $=3.7 \mathrm{~Hz}$ ), 124.1 (app. d, $J=272.5 \mathrm{~Hz}) ;{ }^{19}$ F NMR $(377 \mathrm{MHz}$, $\left.\mathrm{CDCl}_{3}\right) \delta 66.1(\mathrm{~s}, 1 \mathrm{~F}),-62.7(\mathrm{~s}, 3 \mathrm{~F}) ; \mathbf{I R} v_{\max }$ (neat) $/ \mathrm{cm}^{-1} 3076,1619$, 1409, 1325, 1210, 1167, 1125, 1071; LRMS (ESI $\left.{ }^{-}\right) \mathrm{m} / \mathrm{z} 339.1$ $\left([\mathrm{M}+\mathrm{Cl}]^{-}\right)$; HRMS $\left(\mathrm{ESI}^{+}\right) \mathrm{m} / z$ calc. for $\mathrm{C}_{13} \mathrm{H}_{8} \mathrm{~F}_{4} \mathrm{O}_{2} \mathrm{SNa}^{+}[\mathrm{M}+\mathrm{Na}]^{+}$ 327.0073 , found 327.0080 .

\subsubsection{2-(Quinolin-6-yl)benzenesulfonyl fluoride (5n)}

A reaction tube was charged with $\mathrm{KF}(48 \mathrm{mg}, 0.83 \mathrm{mmol}$, 3.3 equiv), $\mathrm{Pd}(\mathrm{OAc})_{2}(11.2 \mathrm{mg}, 0.050 \mathrm{mmol}, 0.20$ equiv) and XPhos (48 mg, $0.10 \mathrm{mmol}, 0.40$ equiv), sealed with a rubber septum, evacuated and back-filled with $\mathrm{N}_{2}$ three times. Degassed anhydrous THF $(0.25 \mathrm{~mL})$ and 6-bromoquinoline $(34 \mu \mathrm{L}$, $0.25 \mathrm{mmol}, 1.0$ equiv) were added subsequently, the mixture was stirred at ambient temperature for $5 \mathrm{~min}$, then warmed to $40^{\circ} \mathrm{C}$ and a solution of 2-(fluorosulfonyl)phenyl boronic acid $3 \mathbf{c}$ (76 mg,
$0.37 \mathrm{mmol}, 1.5$ equiv) in degassed THF ( $1 \mathrm{~mL})$ was added over a period of $30 \mathrm{~min}$. The reaction mixture was stirred at $40{ }^{\circ} \mathrm{C}$ for an additional $30 \mathrm{~min}$, then stirred at ambient temperature for $12 \mathrm{~h}$. The reaction was then diluted with EtOAc, filtered over Celite and concentrated in vacuo. The crude product was purified by flash column chromatography (40-50\% EtOAc in petrol) to afford an off-white solid (30.5 mg), $43 \%$ yield. $\boldsymbol{m p} 95-96{ }^{\circ} \mathrm{C}\left(\mathrm{CHCl}_{3}\right) ; \mathbf{R}_{\boldsymbol{f}}$ $0.31\left(50 \%\right.$ EtOAc in hexane) $\left[\mathrm{KMnO}_{4} / \mathrm{UV}\right] ;{ }^{1} \mathbf{H}$ NMR $(400 \mathrm{MHz}$, $\left.\mathrm{CDCl}_{3}\right) \delta 8.99(\mathrm{dd}, J=4.3,1.7 \mathrm{~Hz}, 1 \mathrm{H}), 8.23(\mathrm{dd}, J=8.1,1.3 \mathrm{~Hz}$, $3 \mathrm{H}), 8.21-8.17(\mathrm{~m}, 2 \mathrm{H}), 7.86-7.84(\mathrm{~m}, 1 \mathrm{H}), 7.81(\mathrm{td}, J=7.6$, $1.4 \mathrm{~Hz}, 1 \mathrm{H}), 7.72(\mathrm{ddd}, J=8.7,2.0,0.9 \mathrm{~Hz}, 1 \mathrm{H}), 7.66(\mathrm{ddt}, J=9.1$, 7.7, $1.4 \mathrm{~Hz}, 1 \mathrm{H}), 7.55(\mathrm{dd}, J=7.7,1.3 \mathrm{~Hz}, 1 \mathrm{H}), 7.47(\mathrm{dd}, J=8.3$, $4.2 \mathrm{~Hz}, 1 \mathrm{H}) ;{ }^{13} \mathbf{C}\left\{{ }^{1} \mathbf{H}\right\}$ NMR $\left(101 \mathrm{MHz}, \mathrm{CDCl}_{3}\right) \delta 151.3,147.9$, $142.3,136.6,136.3,135.0,133.3,132.6(\mathrm{~d}, J=22.4 \mathrm{~Hz}), 130.5$, 130.3, 129.4, 128.7, $128.4, \quad 127.7, \quad 122.0 ; \quad{ }^{19} \mathbf{F}\left\{{ }^{1} \mathbf{H}\right\}$ NMR $\left(377 \mathrm{MHz}, \mathrm{CDCl}_{3}\right) \delta 67.5$; IR $v_{\max }$ (neat) $/ \mathrm{cm}^{-1} 3064,1478,1404$, 1210, 763; LRMS $\left(\mathrm{ESI}^{+}\right) \mathrm{m} / \mathrm{z} 288.0\left([\mathrm{M}+\mathrm{H}]^{+}\right)$; HRMS $\left(\mathrm{ESI}^{+}\right) \mathrm{m} / \mathrm{z}$ calc. for $\mathrm{C}_{15} \mathrm{H}_{11} \mathrm{FNO}_{2} \mathrm{~S}^{+}[\mathrm{M}+\mathrm{H}]^{+}$288.0489, found 288.0488.

4.8. Rh(I)-catalyzed conjugate addition for the synthesis of 4(3-oxocyclohexyl)benzenesulfonyl fluoride (6)

A reaction tube was charged with 4-(fluorosulfonyl)phenylboronic acid $3 a \quad(61 \mathrm{mg}, \quad 0.30 \mathrm{mmol}, 2.0$ equiv), $\left[\mathrm{Rh}(\mathrm{COD})\left(\mathrm{CH}_{3} \mathrm{CN}\right)_{2}\right] \mathrm{BF}_{4} \quad(2.9 \mathrm{mg}, \quad 7.5 \mu \mathrm{mol}, \quad 5.0 \mathrm{~mol} \%)$ and $\mathrm{K}_{2} \mathrm{CO}_{3}$ (21 mg, $0.15 \mathrm{mmol}$, 1.0 equiv), sealed with a rubber septum, evacuated and back-filled with $\mathrm{N}_{2}$ three times. Degassed 1,4-dioxane $(1.35 \mathrm{~mL})$, degassed water $(0.15 \mathrm{~mL})$ and 2cyclohexen-1-one (14.5 $\mu \mathrm{L}, 0.15 \mathrm{mmol}, 1.0$ equiv) were added sequentially, and the reaction mixture stirred under positive pressure of $\mathrm{N}_{2}$ in a preheated aluminium heating block at $55^{\circ} \mathrm{C}$ for $2 \mathrm{~h}$. After cooling to ambient temperature, the reaction mixture was diluted with EtOAc, washed with water and then brine, dried with anhydrous $\mathrm{MgSO}_{4}$, filtered and concentrated in vacuo. The crude product was purified by flash column chromatography (20$40 \%$ EtOAc in petrol) to afford a white solid $(28.5 \mathrm{mg}), 74 \%$ yield. mp $\quad 61-62{ }^{\circ} \mathrm{C}\left(\mathrm{CH}_{2} \mathrm{Cl}_{2}\right) ; \quad \mathbf{R}_{\boldsymbol{f}} 0.38 \quad(30 \%$ EtOAc in hexane $)$ $\left[\mathrm{KMnO}_{4} / \mathrm{UV}\right] ;{ }^{1} \mathbf{H}$ NMR $\left(400 \mathrm{MHz}, \mathrm{CDCl}_{3}\right) \delta 7.98(\mathrm{dt}, J=8.4$, $2.0 \mathrm{~Hz}, 2 \mathrm{H}), 7.48(\mathrm{~d}, J=8.2 \mathrm{~Hz}, 2 \mathrm{H}), 3.15(\mathrm{tt}, J=11.6,4.0 \mathrm{~Hz}$, $1 \mathrm{H}), 2.62$ (ddt, $J=14.0,4.2,1.9 \mathrm{~Hz}, 1 \mathrm{H}), 2.59-2.45(\mathrm{~m}, 2 \mathrm{H}), 2.47-$ $2.34(\mathrm{~m}, 1 \mathrm{H}), 2.25-2.13(\mathrm{~m}, 1 \mathrm{H}), 2.16-2.09(\mathrm{~m}, 1 \mathrm{H}), 1.97-1.74$ $(\mathrm{m}, 2 \mathrm{H}) ;{ }^{13} \mathbf{C}\left\{{ }^{1} \mathbf{H}\right\}$ NMR $\left(101 \mathrm{MHz}, \mathrm{CDCl}_{3}\right) \delta 209.4,152.8,131.5$ $(\mathrm{d}, J=24.7 \mathrm{~Hz}), 129.1,128.1,48.2,44.7,41.1,32.4,25.4$; ${ }^{19} \mathbf{F}\left\{{ }^{1} \mathbf{H}\right\}$ NMR $\left(377 \mathrm{MHz}, \mathrm{CDCl}_{3}\right) \delta 66.1$; IR $v_{\max }$ (neat) $/ \mathrm{cm}^{-1}$ 2980, 2884, 1712, 1597, 1404, 1211, 1186, 804, 771; HRMS $\left(\mathrm{APCI}^{+}\right) \mathrm{m} / z$ calc. for $\mathrm{C}_{12} \mathrm{H}_{14} \mathrm{FO}_{3} \mathrm{~S}^{+}[\mathrm{M}+\mathrm{H}]^{+}$257.0642, found 257.0641 .

\section{Acknowledgements}

We thank the Croucher Foundation (to T.S.-B.L.) for financial support for this research.

\section{Appendix A. Supplementary data}

Supplementary data relating to this article (NMR spectra) may be found at.

\section{References}

1. (a) Bogolubsky, A. V.; Moroz, Y. S.; Mykhailiuk, P. K.; Pipko, S. E.; Konovets, A. I.; Sadkova, I. V.; Tolmachev, A., ACS Comb. Sci. 2014, 16, 192-7; (b) Brouwer, A. J.; Ceylan, T.; Jonker, A. M.; van der Linden, T.; Liskamp, R. M., Bioorg Med Chem 2011, 19, 2397406; (c) Brouwer, A. J.; Jonker, A.; Werkhoven, P.; Kuo, E.; Li, N.; 
Gallastegui, N.; Kemmink, J.; Florea, B. I.; Groll, M.; Overkleeft, H. S.; Liskamp, R. M. J., J. Med. Chem. 2012, 55, 10995-1003.

2. (a) Fadeyi, O.; Parikh, M. D.; Chen, M. Z.; Kyne, R. E., Jr.; Taylor, A. P.; O'Doherty, I.; Kaiser, S. E.; Barbas, S.; Niessen, S.; Shi, M.; Weinrich, S. L.; Kath, J. C.; Jones, L. H.; Robinson, R. P., Chembiochem 2016, 17, 1925-1930; (b) Narayanan, A.; Jones, L. H., Chem. Sci. 2015, 6, 2650-2659; (c) Shannon, D. A.; Gu, C.; McLaughlin, C. J.; Kaiser, M.; van der Hoorn, R. A.; Weerapana, E., Chembiochem 2012, 13, 2327-30; (d) Hett, E. C.; Xu, H.; Geoghegan, K. F.; Gopalsamy, A.; Kyne, R. E., Jr.; Menard, C. A.; Narayanan, A.; Parikh, M. D.; Liu, S.; Roberts, L.; Robinson, R. P.; Tones, M. A.; Jones, L. H., ACS Chem Biol 2015, 10, 1094-8.

3. (a) Dong, J.; Krasnova, L.; Finn, M. G.; Sharpless, K. B., Angew. Chem. Int. Ed. 2014, 53, 9430-48; (b) Barrow, A. S.; Smedley, C. J.; Zheng, Q.; Li, S.; Dong, J.; Moses, J. E., Chem. Soc. Rev. 2019, 48, 4731-4758.

4. Powers, J. C.; Asgian, J. L.; Ekici, Ö. D.; James, K. E., Chem. Rev. 2002, 102, 4639-4750

5. (a) Lively, M. O.; Powers, J. C., Biochimica et Biophysica Acta (BBA) - Enzymology 1978, 525, 171-179; (b) Fahrney, D. E.; Gold, A. M., J. Am. Chem. Soc. 1963, 85, 997-1000.

6. Nielsen, M. K.; Ugaz, C. R.; Li, W.; Doyle, A. G., J. Am. Chem. Soc. 2015, 137, 9571-9574.

7. Bassin, J. P.; Cremlyn, R. J.; Swinbourne, F. J., Phosphorus, Sulfur, and Silicon and the Related Elements 1991, 56, 245-275.

8. Tang, L.; Yang, Y.; Wen, L.; Yang, X.; Wang, Z., Green Chem. 2016, 18, 1224-1228.

9. Emmett, E. J.; Willis, M. C., Organic Syntheses 2014, 91, 125-136. 10. (a) Davies, A. T.; Curto, J. M.; Bagley, S. W.; Willis, M. C., Chem. Sci. 2017, 8, 1233-1237; (b) Tribby, A. L.; Rodriguez, I.; Shariffudin, S.; Ball, N. D., J. Org. Chem. 2017, 82, 2294-2299; (c) Lou, T. S.-B.; Bagley, S. W.; Willis, M. C. Angew. Chem. Int. Ed. 2019, 58 (doi: 10.1002/anie.201910871).

11. Laudadio, G.; Bartolomeu, A. A.; Verwijlen, L.; Cao, Y.; de Oliveira, K. T.; Noel, T., J. Am. Chem. Soc. 2019, 141, 11832-11836. 12. Krutak, J. J.; Burpitt, R. D.; Moore, W. H.; Hyatt, J. A., J. Org. Chem. 1979, 44, 3847-3858.

13. Chinthakindi, P. K.; Arvidsson, P. I., Eur. J. Org. Chem. 2018, 2018, 3648-3666.

14. Zha, G. F.; Zheng, Q.; Leng, J.; Wu, P.; Qin, H. L.; Sharpless, K. B., Angew. Chem. Int. Ed. 2017, 56, 4849-4852.

15. (a) Chinthakindi, P. K.; Govender, K. B.; Kumar, A. S.; Kruger, H. G.; Govender, T.; Naicker, T.; Arvidsson, P. I., Org. Lett. 2017, 19, 480-483; (b) Zha, G.-F.; Bare, G. A. L.; Leng, J.; Shang, Z.-P.; Luo, Z.; Qin, H.-L., Adv. Synth. Catal. 2017, 359, 3237-3242.

16. Qin, H.-L.; Zheng, Q.; Bare, G. A. L.; Wu, P.; Sharpless, K. B., Angew. Chem. Int. Ed. 2016, 55, 14155-14158.

17. (a) Chinthakindi, P. K.; Kruger, H. G.; Govender, T.; Naicker, T.; Arvidsson, P. I., J. Org. Chem. 2016, 81, 2618-23; (b) Cherepakha, A. Y.; Stepannikova, K. O.; Vashchenko, B. V.; Gorichko, M. V.; Tolmachev, A. A.; Grygorenko, O. O., Eur. J. Org. Chem. 2018, 2018, 6682-6692.

18. Steinkopf, W.; Jaeger, P., Journal für Praktische Chemie 1930, $128,63-88$

19. Wang, S.-M.; Li, C.; Leng, J.; Bukhari, S. N. A.; Qin, H.-L., Org. Chem. Front. 2018, 5, 1411-1415.

20. Ishiyama, T.; Murata, M.; Miyaura, N., J. Org. Chem. 1995, 60, 7508-7510.

21. Coutts, S. J.; Adams, J.; Krolikowski, D.; Snow, R. J., Tetrahedron Lett. 1994, 35, 5109-5112.

22. Talko, A.; Antoniak, D.; Barbasiewicz, M., Synthesis 2019, 51, 2278-2286.

23. Knight, S. D.; Adams, N. D.; Burgess, J. L.; Chaudhari, A. M.; Darcy, M. G.; Donatelli, C. A.; Luengo, J. I.; Newlander, K. A.; Parrish, C. A.; Ridgers, L. H.; Sarpong, M. A.; Schmidt, S. J.; Van Aller, G. S.; Carson, J. D.; Diamond, M. A.; Elkins, P. A.; Gardiner, C. M.; Garver, E.; Gilbert, S. A.; Gontarek, R. R.; Jackson, J. R.; Kershner, K. L.; Luo, L.; Raha, K.; Sherk, C. S.; Sung, C. M.; Sutton, D.; Tummino, P. J.; Wegrzyn, R. J.; Auger, K. R.; Dhanak, D., $A C S$ Med Chem Lett 2010, 1, 39-43.
24. Littke, A. F.; Dai, C.; Fu, G. C., J. Am. Chem. Soc. 2000, 122, 4020-4028.

25. (a) Yoshida, K.; Hayashi, T., Rhodium(I)-Catalyzed Asymmetric Addition of Organometallic Reagents to Electron-Deficient Olefins. In Modern Rhodium-Catalyzed Organic Reactions, Wiley-VCH Verlag GmbH \& Co. KGaA: 2005; pp 55-77. (b) Sakai, M.; Hayashi, H.; Miyaura, N. Organometallics 1997, 16, 4229-4231. 